\title{
Large Time Decay and Scattering for Wave Maps
}

\author{
J. Krieger and K. Nakanishi
}

Communicated by Y. Charles Li, received December 21, 2007.

\begin{abstract}
Let $u: \mathbf{R}^{3+1} \longrightarrow \mathbf{H}^{2}$ be a Wave Map with smooth compactly supported initial data satisfying the smallness condition $\|u[0]\|_{\dot{H}^{\frac{3}{2}} \times \dot{H}^{\frac{1}{2}}}<\epsilon$ for a sufficiently small $\epsilon>0$. In particular, the Wave Map exists globally in time and is smooth. Then denoting $u(0, x)=u_{\infty} \in \mathbf{H}$ for $|x|$ large enough, we have

$$
\left\|u(t, x)-u_{\infty}\right\|_{L_{x}^{\infty}} \lesssim t^{-\delta}
$$

for $|t|>>1$ and $\delta>0$ some universal constant. Here the implied constant depends on weighted norms of the the initial data (which, however, need not be small). Furthermore, there exist $(f, g) \in \dot{H}^{\frac{3}{2}} \times \dot{H}^{\frac{1}{2}}$ such that

$$
u(t, x)=S(t)(f, g)+o_{\dot{H}^{\frac{3}{2}}}(1)
$$
\end{abstract}

where $S(t)$ denotes the free wave propagator.

\section{Contents}

1. Introduction 1

2. Wave Maps with target $\mathbf{H}^{2}$ : the basic setup 3

3. Deducing asymptotic decay and scattering from the core estimates 9

4. The core propositions $\quad 15$

References $\quad 36$

\section{Introduction}

A wave map $u: \mathbb{R}^{n+1} \longrightarrow M$ with $(M, g)$ a Riemannian manifold, and $\mathbb{R}^{n+1}$ the $(n+1)$-dimensional Minkowski space with Minkwoski metric $m_{\mu \nu}=\operatorname{diag}(-1,1, \ldots 1)$,

1991 Mathematics Subject Classification. 35.

Key words and phrases. Wave maps, scattering, large time decay. 
is critical with respect to the functional ${ }^{1}$

$$
u \rightarrow \mathcal{L}(u):=\int_{\mathbb{R}^{n+1}}\left\langle\partial_{\alpha} u, \partial^{\alpha} u\right\rangle_{g} d \sigma, \partial^{\alpha}=m^{\alpha \beta} \partial_{\beta}
$$

The Euler Lagrange equations associated with this functional in local coordinate are of the form

$$
\square u^{i}+\Gamma_{j k}^{i}(u) \partial_{\nu} u^{j} \partial^{\nu} u^{k}=0, \square=\partial_{\nu} \partial^{\nu}
$$

Although the global well-posedness of the Wave Maps problem for general smooth large data, generic target, and spatial dimension $n \geq 2$ is far from understood (for a recent survey of results and conjectures see e. g. [12]), global existence results have been obtained with increasingly weaker smallness conditions on the data. The first such results follow from the classical work of Klainerman, which uses vector field methods and in particular imposes a very strong smallness condition on the data, in terms of certain weighted $H^{s}$-norms. These conditions in particular imply that the Wave Map will be confined to a single coordinate chart inside the target. Further, they immediately yield strong asymptotic results for the corresponding solutions, including pointwise decay estimates as well as scattering in terms of the local coordinate functions.

Recent work by Tataru, Tao and others $[\mathbf{2 5}],[\mathbf{2 6}],[\mathbf{2 3}],[\mathbf{2 4}],[\mathbf{6}],[\mathbf{1 3}],[\mathbf{9}],[\mathbf{1 1}],[\mathbf{2 7}]$ based on new techniques introduced in $[\mathbf{2 6}],[\mathbf{2 3}],[\mathbf{2 4}]$, led to significantly stronger global existence results. In particular, one only needs to assume that the initial data $u[0]=\left(u, \partial_{t} u\right)_{t=0}$ are small in the critical Sobolev norm $\|\cdot\|_{\dot{H}^{\frac{n}{2}} \times \dot{H}} \frac{n}{2}-1$ in a suitable sense. Note that the latter condition no longer implies that the data are confined to a single chart: indeed, the Wave Map can now move all over the target. The works $[\mathbf{2 3}],[\mathbf{2 4}],[\mathbf{6}],[\mathbf{1 3}],[\mathbf{9}],[\mathbf{1 1}],[\mathbf{2 7}]$ are based on a geometric trick, introduced in $[\mathbf{2 3}],[\mathbf{2 4}]$, namely exploiting the inherent Gauge invariance of the equations to pass to a more convenient Gauge (e. g. the Coulomb Gauge). Furthermore, they reveal that the cases $n=3$ and especially $n=2$ appear significantly more complicated than the cases $n \geq 4$ since the Strichartz estimates by themselves appear no longer strong enough to close the estimates, even using the inherent nullstructure. Instead, the only method thus far establishing the well-posedness at the critical level hinges on a sophisticated framework from harmonic analysis, blending $X^{s, \theta}$-type or Bourgain spaces with Tataru's null-frame spaces, both of which are based on localizations of the space-time Fourier transform of the unknown function. Both the fact that one needs to pass into a new Gauge as well as the use of complex spaces based on the space-time Fourier transform render the question of global asymptotic behavior as well as scattering in the original coordinates highly non-trivial in the dimensions $n=2,3$. The present paper has the goal of answering these questions for $3+1$-dimensional ${ }^{2}$ Wave Maps with target $\mathbf{H}^{2}$, the hyperbolic plane. This target appears quite natural, as it occurs in the context of General Relativity, and is the natural counterpoint to Wave Maps with target $S^{2}$. As already observed in [9], and exploited in [11], Wave Maps with target $\mathbf{H}^{2}$ enjoy the remarkable property that the derivative components in the Coulomb Gauge satisfy an autonomous first order div-curl system, which no longer involves the local coordinate functions.

\footnotetext{
${ }^{1}$ Throughout this paper, the Einstein summation convention is in force. This means that we sum over repeated raised and lowered indices.

${ }^{2}$ Our argument can obviously be modified to also handle the higher dimensional cases $n \geq$ 4. Indeed, the argument becomes much simpler then, since one has stronger estimates for the nonlinearity.
} 
This allows one to conveniently disentangle the global behavior of these derivative components from the global behavior of the actual coordinate functions, and to pass from the former to the latter. The main result of this paper is the following

Theorem 1.1. Let $u: \mathbf{R}^{3+1} \longrightarrow \mathbf{H}^{2}$ be a Wave Map with smooth compactly supported ${ }^{3}$ initial data satisfying the smallness condition (suitably interpreted as explained below) $\|u[0]\|_{\dot{H}^{\frac{3}{2}} \times \dot{H}^{\frac{1}{2}}}<\epsilon$ for a sufficiently small $\epsilon>0$. In particular, the Wave Map exists globally in time and is smooth. Then denoting $u(0, x)=u_{\infty} \in \mathbf{H}$ for $|x|$ large enough, we have

$$
\left\|u(t, x)-u_{\infty}\right\|_{L_{x}^{\infty}} \lesssim t^{-\delta}
$$

for $|t|>>1$ and $\delta>0$ some universal constant. Here the implied constant depends on weighted norms of the the initial data (which, however, need not be small). Furthermore, there exist $(f, g) \in \dot{H}^{\frac{3}{2}} \times \dot{H}^{\frac{1}{2}}$ such that

$$
u(t, x)=S(t)(f, g)+o_{\dot{H}^{\frac{3}{2}}}(1)
$$

where $S(t)$ denotes the free wave propagator.

REMARK 1.2. The critical case $n=2$ appears much more technically involved, although the same strategy should work in principle, see the estimates in [11]. Furthermore, a similar method should in principle work for more general targets, although the fact that one can no longer formulate an autonomous system of wave equations for the derivative components (they now also depend on the local coordinates) introduces additional technical difficulties.

\section{Wave Maps with target $\mathbf{H}^{2}$ : the basic setup}

We use the setup in [11] : identify $\mathbf{H}^{2}=\{(\mathbf{x}, \mathbf{y}) \mid \mathbf{y} \geq 0\}$ equipped with metric $d g=\frac{d \mathbf{x}^{2}+d \mathbf{y}^{2}}{\mathbf{y}^{2}}$. Then introduce the derivative components $\phi_{\alpha}^{1}=\frac{\partial_{\alpha} \mathbf{x}}{\mathbf{y}}, \phi_{\alpha}^{2}=\frac{\partial_{\alpha} \mathbf{y}}{\mathbf{y}}$, $\alpha=0,1,2,3$. Further, introduce the derivative components in the Coulomb Gauge in complex notation

$$
\psi_{\alpha}=\psi_{\alpha}^{1}+i \psi_{\alpha}^{2}=\left(\phi_{\alpha}^{1}+i \phi_{\alpha}^{2}\right) e^{-i \triangle^{-1} \sum_{j=1}^{3} \partial_{j} \phi_{j}^{1}}
$$

We recall that we then obtain the following divergence curl system

$$
\begin{gathered}
\partial_{\alpha} \psi_{\beta}-\partial_{\beta} \psi_{\alpha}=i \psi_{\beta} \triangle^{-1} \sum_{j=1}^{3} \partial_{j}\left(\psi_{\alpha}^{1} \psi_{j}^{2}-\psi_{\alpha}^{2} \psi_{j}^{1}\right)-i \psi_{\alpha} \triangle^{-1} \sum_{j=1}^{3} \partial_{j}\left(\psi_{\beta}^{1} \psi_{j}^{2}-\psi_{\beta}^{2} \psi_{j}^{1}\right) \\
\partial^{\nu} \psi_{\nu}=i \psi_{\nu} \triangle^{-1} \sum_{j=1}^{3} \partial_{j}\left(\psi_{\nu}^{1} \psi_{j}^{2}-\psi_{\nu}^{2} \psi_{j}^{1}\right)
\end{gathered}
$$

\footnotetext{
${ }^{3}$ This means that $u(0, x)=u_{\infty}$ is a fixed point for $|x|$ sufficiently large, as well as $\partial_{t} u(0, x)=$ 0 . One may weaken this condition to sufficiently fast decay at infinity.
} 
From here one infers the wave equations

$$
\begin{aligned}
\square \psi_{\alpha}= & i \partial^{\beta}\left[\psi_{\alpha} \triangle^{-1} \sum_{j=1}^{3} \partial_{j}\left(\psi_{\beta}^{1} \psi_{j}^{2}-\psi_{\beta}^{2} \psi_{j}^{1}\right)\right] \\
& -i \partial^{\beta}\left[\psi_{\beta} \triangle^{-1} \sum_{j=1}^{3} \partial_{j}\left(\psi_{\alpha}^{1} \psi_{j}^{2}-\psi_{\alpha}^{2} \psi_{j}^{1}\right)\right] \\
& +i \partial_{\alpha}\left[\psi_{\nu} \triangle^{-1} \sum_{j=1}^{3} \partial_{j}\left(\psi^{1 \nu} \psi_{j}^{2}-\psi^{2 \nu} \psi_{j}^{1}\right)\right]
\end{aligned}
$$

Schematically speaking, the terms on the right are of the form $\nabla_{x, t}\left[\psi \nabla^{-1}\left[\psi^{2}\right]\right]$. In dimensions $n=2,3$, the right hand side of (2.3) is not amenable to estimates as is, and as in [11], we need to exploit the underlying divergence-curl structure to decompose it into a null-form plus a better error term. For this, we split

$$
\psi_{\alpha}=R_{\alpha} \psi+\chi_{\alpha}, \alpha=0,1,2,3,
$$

where we impose the vanishing divergence condition $\sum_{j=1}^{3} \partial_{j} \chi_{j}=0$. Here, the symbols $R_{\alpha}=\nabla^{-1} \partial_{\alpha}$ with $\widehat{\nabla^{-1} f}(\xi)=|\xi|^{-1} \hat{f}(\xi)$ denote Riesz type operators. The $\chi_{\alpha}$ can then be obtained as solutions of an elliptic divergence curl system, and are schematically of the form

$$
\chi_{\alpha}=\nabla^{-1}\left[\psi \nabla^{-1}\left(\psi^{2}\right)\right]
$$

If we now insert the splitting (2.4) into (2.3), we can replace the right hand side by

$$
\begin{aligned}
\square \psi_{\alpha}= & i \partial^{\beta}\left[R_{\alpha} \psi \triangle^{-1} \sum_{j=1}^{3} \partial_{j}\left(R_{\beta} \psi^{1} R_{j} \psi^{2}-R_{\beta} \psi^{2} R_{j} \psi^{1}\right)\right] \\
& -i \partial^{\beta}\left[R_{\beta} \psi \triangle^{-1} \sum_{j=1}^{3} \partial_{j}\left(R_{\alpha} \psi^{1} R_{j} \psi^{2}-R_{\alpha} \psi^{2} R_{j} \psi^{1}\right)\right] \\
& +i \partial_{\alpha}\left[R_{\nu} \psi \triangle^{-1} \sum_{j=1}^{3} \partial_{j}\left(R^{\nu} \psi^{1} R_{j} \psi^{2}-R^{\nu} \psi^{2} R_{j} \psi^{1}\right)\right] \\
& +\nabla_{x, t}\left[\nabla^{-1}\left[\psi \nabla^{-1}\left(\psi^{2}\right)\right] \nabla^{-1}\left(\psi^{2}\right)\right]+\nabla_{x, t}\left[\psi \nabla^{-1}\left[\nabla^{-1}\left[\psi \nabla^{-1}\left(\psi^{2}\right)\right] \psi\right]\right] \\
& +\nabla_{x, t}\left[\psi \nabla^{-1}\left[\nabla^{-1}\left[\psi \nabla^{-1}\left(\psi^{2}\right)\right] \nabla^{-1}\left[\psi \nabla^{-1}\left(\psi^{2}\right)\right]\right]\right]
\end{aligned}
$$

Here the last three expressions are of course recorded schematically, with each $\nabla^{-1}$ denoting operators of the form $\sum_{k=1}^{3} \triangle^{-1} \partial_{k}$. It is this complicated system of wave equations which shall be at the heart of our analysis, similarly to [11].

Recall that the basic paradigm for establishing scattering for a wave equation of the form

$$
\square u=F(u, \nabla u)
$$

is to establish $\lim _{|t| \rightarrow \infty} \int_{t}^{\infty} U(t-s) F(u, \nabla u)(s) d s=0$ in the underlying Sobolev space $\dot{H}^{s}$ (which is dictated by scaling reasons, for example). This follows by establishing $L_{t}^{1} \dot{H}^{s}$-bounds on the source $F(u, \nabla u)$. For our system $(2.3)$, such estimates are not available in dimensions $n=2,3$. 
2.1. The method to obtain scattering for the $\psi_{\alpha}$. Fixing a large dyadic time interval $I_{l}:=\left[-2^{l+2}, 2^{l+2}\right], l>>1$, we shall split the components $\psi_{\alpha}$ into two parts $\psi_{\alpha}=\psi_{\alpha, 1}+\psi_{\alpha, 2}$. Here $\psi_{\alpha, 1}$ approximates the moderate-frequency part of $\psi_{\alpha}$ (where 'moderate' is in relation to the scale $2^{l}$ ), while $\psi_{\alpha, 2}$ approximates the very large/small frequency part of $\psi_{\alpha, 2}$. While $\psi_{\alpha, 2}$ will turn out to be small since the extreme frequencies of $\psi_{\alpha}$ carry only little energy, $\psi_{\alpha, 1}$ will be shown to be small (albeit in a different sense) since we shall be able to apply commutating vector fields to it. Thus the simple basic premise of the present approach is to split $\psi_{\alpha}$ into a moderate frequency part, obtained by filtering out the extreme frequencies in the nonlinearity, which is basically amenable to classical commutating vector field methods (although of course $\psi_{\alpha, 1}$ is not small with respect to the weighted norms), as well as an error term which accounts for the remaining errors due to extreme frequencies. Scattering of $\psi_{\alpha}$ will then follow by splitting the right hand side of (2.3) into two parts, upon writing $\psi_{\alpha}=\psi_{\alpha, 1}+\psi_{\alpha, 2}$. Indeed, crudely denoting the right hand side of $(2.3)$ as $F(\psi)$ (it being understood that it is not a locally defined function evaluated at $\psi$ ), we can decompose

$$
F\left(\psi_{\alpha}\right)=\sum_{l} \chi_{t \sim 2^{l}}\left[F\left(\psi_{\alpha, 1}\right)+\text { error }\right]
$$

where the splitting $\psi_{\alpha}=\psi_{\alpha, 1}+\psi_{\alpha, 2}$ is the one on the interval $\left[-2^{l+2}, 2^{l+2}\right]$. We shall then show that $\psi_{\alpha, 1}$ can be placed into $L_{t}^{2} L_{x}^{\infty}$, which allows us to estimate $F\left(\psi_{\alpha, 1}\right)$ in $L_{1}^{1} \dot{H}^{-\frac{1}{2}}$, while the error is exponentially decaying in $l$ (but with respect to a more complicated norm!). This shall then imply scattering for $\psi_{\alpha}$, as well as pointwise decay. The latter facts allow one to retrace the steps from local coordinates to the $\psi_{\alpha}$ to obtain decay and scattering for the coordinates $(\mathbf{x}, \mathbf{y})$.

2.2. Tools from harmonic analysis. In order to precisely define $\psi_{\alpha, 1}$, we introduce the Littlewood-Paley localizers $P_{k}, k \in \mathbb{Z}$, as well as the space-time localizers $Q_{k}$, as follows: choose a function $\phi \in C_{0}^{\infty}\left(\mathbb{R}_{+}\right)$with the property $\sum_{j \in \mathbb{Z}} \phi\left(\frac{x}{2^{j}}\right)=$ 1, $x>0$. See e.g. [20]. The we define $P_{k}, Q_{k}$ via

$$
\widehat{P_{k} f}(\xi)=\phi\left(\frac{\xi}{2^{k}}\right) \hat{f}(\xi), \widetilde{Q_{k} f}(\tau, \xi)=\phi\left(\frac{|| \tau|-| \xi||}{2^{k}}\right) \tilde{f}(\tau, \xi)
$$

Here ^ denotes the spatial Fourier transform $\hat{f}(\xi)=\int_{\mathbb{R}^{3}} f(x) e^{-2 \pi i x \cdot \xi} d x$, while we denote the space-time Fourier transform by $\tilde{f}(\tau, \xi)=\int_{\mathbb{R}^{3+1}} f(t, x) e^{-2 \pi i(t \tau+x \cdot \xi)} d t d x$. We can then also introduce the operators

$$
P_{<l}=\sum_{k<l} P_{k}, P_{[a, b]}=\sum_{k \in[a, b]} P_{k}, \text { etc }
$$

Further, let $F(\psi)$ denote any of the multilinear expressions on the right hand side of (2.3). Then introduce the operator $\mathbf{P}_{[-\delta l, \delta l]}$, which acts by restricting the frequencies and modulations (i. e. the distance of the space-time Fourier support to the light cone, measured by ||$\tau|-| \xi||)$ of all the inputs, as follows:

Definition: the function $\mathbf{P}_{[-\delta l, \delta l]} F(\psi)$ is obtained from $F(\psi)$ by

(i) replacing the ith input $\psi$ by $P_{k_{i}} Q_{<k_{i}+\delta l} \psi$, and summing over $k_{i} \in[-\delta l, \delta l]$ for each $i$.

(ii) replacing the ith operator $\triangle^{-1} \partial_{j}$ by $P_{r_{i}} \triangle^{-1} \partial_{j}$ and summing over $r_{i} \in[-\delta l, \delta l]$ for each $i$. 
(iii) Applying an operator $P_{k} Q_{<k+\delta l}$ to the output and summing over $k \in[-\delta l, \delta l]$.

Example: the trilinear expression

$$
i \partial^{\beta}\left[\psi_{\alpha} \triangle^{-1} \sum_{j=1}^{3} \partial_{j}\left(\psi_{\beta}^{1} \psi_{j}^{2}-\psi_{\beta}^{2} \psi_{j}^{1}\right)\right]
$$

is replaced by

$$
\begin{aligned}
& \mathbf{P}_{[-\delta l, \delta l]}\left[i \partial^{\beta}\left[\psi_{\alpha} \triangle^{-1} \sum_{j=1}^{3} \partial_{j}\left(\psi_{\beta}^{1} \psi_{j}^{2}-\psi_{\beta}^{2} \psi_{j}^{1}\right)\right]\right] \\
& =\sum_{k, k_{1,2,3}, r \in[-\delta l, \delta l]} P_{k} Q_{<k+\delta l}\left[i \partial ^ { \beta } \left[P_{k_{1}} Q_{<k_{1}+\delta l} \psi_{\alpha} \triangle^{-1} P_{r}\right.\right. \\
& \sum_{j=1}^{3} \partial_{j}\left(P_{k_{2}} Q_{<k_{2}+\delta l} \psi_{\beta}^{1} P_{k_{3}} Q_{<k_{3}+\delta l} \psi_{j}^{2}\right. \\
& \left.\left.-P_{k_{3}} Q_{<k_{3}+\delta l} \psi_{\beta}^{2} P_{k_{2}} Q_{<k_{2}+\delta l} \psi_{j}^{1}\right)\right]
\end{aligned}
$$

2.3. Definition of $\psi_{\alpha, 1}, \psi_{\alpha, 2}$ on some interval $\left[-2^{l+2}, 2^{l+2}\right]$. We can now define the equation which defines $\psi_{\alpha, 1}$, as follows:

$$
\begin{aligned}
\square \psi_{\alpha, 1}= & \mathbf{P}_{[-\delta l, \delta l]}\left[i \partial^{\beta}\left[R_{\alpha} \psi_{1} \triangle^{-1} \sum_{j=1}^{3} \partial_{j}\left(R_{\beta} \psi_{1}^{1} R_{j} \psi_{1}^{2}-R_{\beta} \psi_{1}^{2} R_{j} \psi_{1}^{1}\right)\right]\right. \\
& -i \partial^{\beta}\left[R_{\beta} \psi_{1} \triangle^{-1} \sum_{j=1}^{3} \partial_{j}\left(R_{\alpha} \psi_{1}^{1} R_{j} \psi_{1}^{2}-R_{\alpha} \psi_{1}^{2} R_{j} \psi_{1}^{1}\right)\right] \\
& +i \partial_{\alpha}\left[R_{\nu} \psi_{1} \triangle^{-1} \sum_{j=1}^{3} \partial_{j}\left(R^{\nu} \psi_{1}^{1} R_{j} \psi_{1}^{2}-R^{\nu} \psi_{1}^{2} R_{j} \psi_{1}^{1}\right)\right] \\
& +\nabla_{x, t}\left[\nabla^{-1}\left[\psi_{1} \nabla^{-1}\left(\psi_{1}^{2}\right)\right] \nabla^{-1}\left(\psi_{1}^{2}\right)\right]+\nabla_{x, t}\left[\psi_{1} \nabla^{-1}\left[\nabla^{-1}\left[\psi_{1} \nabla^{-1}\left(\psi_{1}^{2}\right)\right] \psi_{1}\right]\right] \\
& \left.+\nabla_{x, t}\left[\psi_{1} \nabla^{-1}\left[\nabla^{-1}\left[\psi_{1} \nabla^{-1}\left(\psi_{1}^{2}\right)\right] \nabla^{-1}\left[\psi_{1} \nabla^{-1}\left(\psi_{1}^{2}\right)\right]\right]\right]\right] \\
& \psi_{\alpha, 1}[0]=\psi_{\alpha}[0]:=\left(\psi_{\alpha}(0), \partial_{t} \psi_{\alpha}(0)\right)
\end{aligned}
$$

Here we use as before $\psi_{1}=-\sum_{j=1}^{3} R_{j} \psi_{j, 1}$ for the first three trilinear terms on the right. For the schematic higher order terms, it is understood that $\psi_{\alpha}$ is replaced by $\psi_{\alpha, 1} \forall \alpha$.

We immediately observe that this is not a standard wave equation, since it involves nonlocal operators in its source term, even in the space-time sense. Thus it certainly does not satisfy Huyghen's principle! Nevertheless, we shall be able to construct solutions on some interval $[-T, T]$ which contains $\left[-2^{l+2}, 2^{l+2}\right]$ and also matches the initial data, via Banach iteration in a suitable space. Indeed, the solution will be smooth. We note here that this iteration is qualitatively different than the procedure used in [11]. There the equation (2.3) is only used to deduce $a$ priori estimates, while the local existence of a solution is ensured by the classical local existence theory in local coordinates. For the problem (2.6), such a step is not possible, since it is not a geometrically motivated problem. Thus the existence 
of local solutions needs to be demonstrated from scratch via iteration.

We can now also define $\psi_{\alpha, 2}$ via

$$
\psi_{\alpha, 2}=\psi_{\alpha}-\psi_{\alpha, 1}
$$

2.4. Analytical preliminaries. Here we recall the functional framework underpinning [26], [24], [11], in Tao's formulation. We shall use the following homogeneous $X^{s, \theta}$-type norm:

$$
\|\psi\|_{\dot{X}_{k}^{p, q, r}}:=2^{k p}\left(\sum_{j \in \mathbb{Z}}\left[2^{q j}\left\|Q_{j} \psi\right\|_{L_{t}^{2} L_{x}^{2}}\right]^{r}\right)^{\frac{1}{r}},
$$

provided $r<\infty$, as well as the obvious modification when $r=\infty$. In order to estimate the frequency localized components of $\psi_{\alpha}$, we have the family of norms $\|\cdot\|_{S[k]}, k \in \mathbb{Z}$, defined as follows: let

$$
\begin{aligned}
\|\psi\|_{S[k]}:= & \left.\left\|\nabla_{x, t} \psi\right\|\right|_{L_{t}^{\infty} \dot{H}^{-\frac{1}{2}}}+\left\|\nabla_{x, t} \psi\right\|_{\dot{X}_{k}^{-\frac{1}{2}, \frac{1}{2}, \infty}}+ \\
& \sup _{l<-10}\left(\sum_{\kappa \in K_{l}}\left\|P_{k, \pm \kappa} Q_{<k+2 l}^{ \pm} \psi\right\|_{S[k, \pm \kappa]}^{2}\right)^{\frac{1}{2}}
\end{aligned}
$$

Here we have chosen for each integer $l<-10$ a finitely overlapping cover $K_{l}$ (with the overlapping being uniform in $l$ ) of caps $\kappa$ of size $\sim 2^{l}$ of the sphere $S^{2}$. The superscript \pm in $Q_{<k+2 l}^{ \pm}$indicates that we further localize (sharply) to the upper or lower half-space $\pm \tau>0$, repsectively, and finally, the norms $\|.\|_{S[k, \kappa]}$ are defined as follows: first, let

$$
\|\psi\|_{N F A[\kappa]^{*}}:=\sup _{\omega \notin 2 \kappa} \operatorname{dist}(\omega, \kappa)\|\psi\|_{L_{t_{\omega}}^{\infty} L_{x_{\omega}}^{2}}
$$

where $\omega$ ranges over $S^{2}$ and we use the null-coordinates

$$
t_{\omega}:=(t, x) \cdot \frac{1}{\sqrt{2}}(1, \omega), x_{\omega}=(t, x)-t_{\omega} \frac{1}{\sqrt{2}}(1, \omega)
$$

We then also have the dual norm, i. e.

$$
\|\psi\|_{N F A[\kappa]}:=\inf _{\int_{\omega \in S^{2}} \psi_{\omega}=\psi} \int_{\omega \notin 2 \kappa} \frac{1}{\operatorname{dist}(\omega, \kappa)}\left\|\psi_{\omega}\right\|_{L_{t_{\omega}}^{1} L_{x_{\omega}}^{2}}
$$

Further, we introduce

$$
\|\psi\|_{P W[\kappa]}:=\inf _{\int_{\omega \in \kappa} \psi_{\omega}=\psi} \int_{\omega \in \kappa}\left\|\psi_{\omega}\right\|_{L_{t_{\omega}}^{2} L_{x_{\omega}}^{\infty}}
$$

Then we put

$$
\|\psi\|_{S[k, \kappa]}:=2^{\frac{k}{2}}\|\psi\|_{N F A[\kappa]^{*}}+|\kappa|^{-\frac{1}{2}} 2^{-\frac{k}{2}}\|\psi\|_{P W[\kappa]}+2^{\frac{k}{2}}\|\psi\|_{L_{t}^{\infty} L_{x}^{2}}
$$

Further, the frequency localized components of the source term, i. e. the right hand side of (2.3) etc, shall be evaluated with respect to the following norm:

$$
\begin{aligned}
\|\psi\|_{N[k]}:= & \inf _{\psi_{1}+\psi_{2}+\psi_{3}+\psi_{4}=P_{k} Q_{<k+10} \psi}\left\|\psi_{1}\right\|_{L_{t}^{1} \dot{H}^{-\frac{1}{2}}}+\left\|\psi_{2}\right\|_{\dot{X}^{-\frac{1}{2},-\frac{1}{2}, 1}} \\
& +\inf _{\sum_{l} \psi_{3 l}=\psi_{3}}\left(\inf _{\sum_{\kappa \in K_{l}}^{\prime} \psi_{\kappa}=\psi_{3 l}} \sum_{\kappa \in K_{l}}\left\|\psi_{\kappa}\right\|_{N F A[\kappa]}^{2}\right)^{\frac{1}{2}} \\
& +\left\|P_{k} Q_{>k+10} \psi\right\|_{\dot{X}^{-\frac{1}{2},-\frac{1}{2}, \infty} \cap \square\left|\nabla_{x, t}\right|^{-1} L_{t}^{\infty} L_{x}^{-\frac{1}{2}}}
\end{aligned}
$$

Here in the last term but one upon fixing $l<-10$ we only consider those $\psi_{\kappa}$ with Fourier satisfying ||$\tau|-| \xi|| \leq 2^{k-2 l-100}, 2^{k-4} \leq|\xi| \leq 2^{k+4}, \Theta:=\frac{\xi}{|\xi|} \frac{\tau}{|\tau|} \in \frac{1}{2} \kappa$. We 
note that the norm $\|\cdot\|_{N[k]}$ we use here is slightly different than the one in [11], as the elliptic portion $P_{k} Q_{>k+10} \psi$ is estimate with a weaker norm, namely

$$
\left\|P_{k} Q_{>k+10} \psi||_{\dot{X}_{k}^{-\frac{1}{2},-\frac{1}{2}, \infty}}+\right\| \nabla_{x, t} \square^{-1} P_{k} Q_{>k+10} \psi \|_{L_{t}^{\infty} \dot{H}_{x}^{-\frac{1}{2}}}
$$

This change barely affects the estimates, though.

Finally, we need time localized versions of these norms: for $T>0$, we introduce

$$
\|\psi\|_{N[k]\left([-T, T] \times \mathbb{R}^{3}\right)}:=\inf _{f \in \mathcal{S}\left(\mathbb{R}^{3+1}\right)|f|_{[-T, T] \times \mathbb{R}^{3}}=\left.\psi\right|_{[-T, T] \times \mathbb{R}^{3}}}\|f\|_{N[k]}
$$

and similarly for $\|\cdot\|_{N[k]\left([-T, T] \times \mathbb{R}^{3}\right)}$ etc.

2.5. Some geometric preliminaries. Here we quickly recall the infinitesimal generators of the Poincare group on $\mathbb{R}^{3+1}$, namely the vector fields $\Gamma$ which are given by $\partial_{t}, \partial_{x_{i}}, i=1,2,3$ (translations), $t \partial_{t}+\sum_{i=1}^{3} x_{i} \partial_{x_{i}}$ (scaling), $x_{i} \partial_{x_{j}}-x_{j} \partial_{x_{i}}$, $i, j,=1,2,3$ (rotations), $t \partial_{x_{i}}+x_{i} \partial_{t}, i=1,2,3$ (Lorentz boosts). We shall denote these by $\Gamma^{\alpha}$ where $\alpha=1,2, \ldots, 11$ for some ordering. We shall denote products of these by $\Gamma^{\alpha_{1}} \Gamma^{\alpha_{2}}=: \Gamma^{\left(\alpha_{1}, \alpha_{2}\right)}$. In particular, the notation $\Gamma^{\beta},|\beta|=2$, refers to a product of two such vector fields (as an operator, with $\beta$ a pair of indices, hence of length two), while $\Gamma^{\beta},|\beta|=1$, refers to a single such vector field. We recall that we then have the relations

$$
\begin{gathered}
{\left[\square, \Gamma^{\beta}\right]=c_{\beta} \square,|\beta|=1} \\
{\left[\square, \Gamma^{\beta}\right]=c_{\beta \gamma} \Gamma^{\gamma} \square+d_{\beta} \square,|\beta|=2,|\gamma|=1}
\end{gathered}
$$

2.6. The core estimates. With the above setup, we can now formulate

Proposition 2.1. Make the same assumptions as in Theorem 1.1. Specifically, we assume that the coordinate functions at time zero $(\mathbf{x}, \mathbf{y})(0, x): \mathbb{R}^{3} \longrightarrow\left(\mathbb{R} \times \mathbb{R}_{+}\right)$, $\left(\partial_{t} \mathbf{x}, \partial_{t} \mathbf{y}\right)(0, x): \mathbb{R}^{3} \longrightarrow(\mathbb{R} \times \mathbb{R})$ satisfy the condition

$$
\int_{\mathbb{R}^{3}}\left[\left(\mid \frac{\nabla_{x} \mathbf{x} \mid}{\mathbf{y}}\right)^{2}+\left(\frac{\left|\nabla_{x} \mathbf{x}\right|}{\mathbf{y}}\right)^{2}\right] d x+\int_{\mathbb{R}^{3}}\left[\left(\frac{\partial_{t} \mathbf{x}}{\mathbf{y}}\right)^{2}+\left(\frac{\partial_{t} \mathbf{x}}{\mathbf{y}}\right)^{2}\right] d x<\epsilon
$$

for sufficiently small $\epsilon>0$. Further, fix a time scale $2^{l}, l>>1$. Then there exists a smooth solution $\psi_{\alpha, 1}$ solving (2.6) on $\left[-2^{l+2}, 2^{l+2}\right]$. Further, the following estimates hold: Introducing the families of numbers

$$
\begin{gathered}
c_{k}:=\sum_{0 \leq|\beta| \leq 3}\left(\sum_{l \in \mathbb{Z}} 2^{-\sigma|k-l|}\left\|P_{l} \Gamma^{\beta} \psi[0]\right\|_{\dot{H}^{\frac{1}{2}} \times \dot{H}^{-\frac{1}{2}}}^{2}\right)^{\frac{1}{2}}, \\
\left.d_{k}:=\sum_{l \in \mathbb{Z}} 2^{-\sigma|l-k|} \|\left. P_{l} \psi[0]\right|_{\dot{H}^{\frac{1}{2}} \times \dot{H}^{-\frac{1}{2}}} ^{2}\right)^{\frac{1}{2}}
\end{gathered}
$$

for a sufficiently small $\sigma>0$, we have

$$
\sum_{0 \leq|\beta| \leq 2}\left\|P_{k} \Gamma^{\beta} \psi_{\alpha, 1}\right\|_{S[k]} \lesssim 2^{C \delta l} c_{k}
$$

for some fixed $C$. We also have

$$
\left\|P_{k} \psi_{\alpha, 1}\right\|_{S[k]} \lesssim d_{k}
$$

Proposition 2.2. Under the same assumptions as in the previous Proposition, we have

$$
\left\|P_{k} \psi_{\alpha, 2}\right\|_{S[k]} \lesssim \min \left\{d_{k}, 2^{\mu \max \{k-\delta l,-k-\delta l\}} d_{k}\right\}:=e_{k}
$$

for a sufficiently small $\mu>0$. 
These Propositions will follow essentially from estimates established in [9], [10], as well as some elementary observations concerning commutators of the $\Gamma^{\beta}$ and Fourier localizers. They will be deferred to the end of the paper. In a similar vein, we have the following

Proposition 2.3. Let $F(\psi)$ denote the right hand side of (2.3). Then for $T \sim 2^{l}$ we have

$$
\left\|P_{k}\left(1-\mathbf{P}_{[-\delta l, \delta l]}\right) F(\psi)\right\|_{N[k]\left([-T, T] \times \mathbb{R}^{3}\right)} \lesssim e_{k}
$$

where $e_{k}$ is as in Proposition 2.2. The same estimate applies if we replace $\psi$ by $\psi_{1}$ (i. e. we replace each $\psi_{\alpha}$ by $\psi_{\alpha, 1}$ ). Also, without any further localization, we have

$$
\left\|P_{k} F\left(\psi_{1}, \psi_{2}\right)\right\|_{N[k]\left([-T, T] \times \mathbb{R}^{3}\right)} \lesssim e_{k}
$$

where $F\left(\psi_{1}, \psi_{2}\right)$ is any of the multilinear expressions on the right hand side of (2.3) with at least one factor $\psi_{\alpha, \nu, \beta}$ replaced by $\psi_{\alpha, \nu, \beta, 2}$.

REMARK 2.4. The numbers $d_{k}, e_{k}$ form frequency envelopes, a notation borrowed from [23]. A frequency envelope is a sequence $\left\{c_{k}\right\}_{k \in Z}$ of non-negative numbers such that for some $\sigma>0$ we have

$$
2^{-\sigma|k-l|} c_{l} \leq c_{k} \leq 2^{\sigma|k-l|} c_{l}
$$

for each $k, l$.

\section{Deducing asymptotic decay and scattering from the core estimates}

Assuming the above Propositions, we now deduce asymptotic decay for the $\psi_{\alpha, 1}, \psi_{\alpha, 2}$, as well as scattering for $\psi_{\alpha}$. Combining these ingredients, we then obtain Theorem 1.1. We commence with the following crucial

LEMmA 3.1. Let $\psi_{\alpha, 1}$ be as above, and assume $t \in\left[-2^{l+2}, 2^{l+2}\right]$ satisfies $t \sim 2^{l}$. Then we have

$$
\left|\psi_{\alpha, 1}(t, x)\right| \lesssim 2^{C \delta l-\frac{2}{3} l}
$$

In particular, if we choose $\delta$ small enough, we can achieve $\left|\psi_{\alpha, 1}(t, x)\right| \lesssim 2^{-\frac{7 l}{12}}$.

Proof. (Lemma 3.1) This is a consequence of the Klainerman-Sobolev inequalities: recall (see e. g. $[\mathbf{2 2}])$ that we have

$$
\left|\psi_{\alpha, 1}(t, x)\right| \lesssim(1+|t|+|x|)^{-1}(1+|| t|-| x||)^{-\frac{1}{2}} \sum_{|\beta| \leq 2}\left\|\Gamma^{\beta} \psi_{\alpha, 1}\right\|_{L_{x}^{2}}
$$

Assume first that $|x| \gtrsim 2^{l}$. Using a smooth cutoff function $\chi_{|| t|-| x|| \sim 2^{s}}$, we localize this to

$$
\begin{aligned}
& \left|\chi_{|x| \gtrsim 2^{l}} \chi_{|| t|-| x|| \sim 2^{s}} \psi_{\alpha, 1}(t, x)\right| \lesssim(1+|t|+|x|)^{-1}(1+|| t|-| x||)^{-\frac{1}{2}} \\
& \times \sum_{|\beta| \leq 2}\left\|\Gamma^{\beta}\left[\chi_{|x| \gtrsim^{l}} \chi_{|| t|-| x|| \sim 2^{s}} \psi_{\alpha, 1}\right]\right\|_{L_{x}^{2}}
\end{aligned}
$$

for $s=1,2,3, \ldots$ It is straightforward to verify that the expressions

$$
\Gamma^{\beta}\left[\chi_{|x| \gtrsim 2^{l}} \chi_{|t|-|x| \mid \sim 2^{s}}\right],|\beta| \leq 2,
$$


are uniformly bounded for $|x| \gtrsim 2^{l}$. Now we distinguish between the cases $2^{s}>>$ $|t| \sim 2^{l}, 2^{s} \lesssim|t| \sim 2^{l}$. In the former, we have using Proposition 2.1 as well as the Sobolev embedding and Holder's inequality

$$
\begin{aligned}
& \left\|\Gamma^{\beta}\left[\chi_{|x| \gtrsim^{l}} \chi_{|| t|-| x|| \sim 2^{s}} \psi_{\alpha, 1}\right]\right\|_{L_{x}^{2}} \\
& \lesssim\left\|\Gamma^{\beta}\left[\chi_{|x| \gtrsim^{l}} \chi_{|| t|-| x|| \sim 2^{s}} \psi_{\alpha, 1}\right]\right\|_{\dot{H}_{x}^{\frac{1}{2}}}\left\|\chi_{|| t|-| x|| \sim 2^{s}} \chi_{|t| \sim 2^{l}}\right\|_{L_{x}^{6}} \lesssim 2^{C \delta l} 2^{\frac{s}{2}},
\end{aligned}
$$

whence we obtain

$$
\left|\chi_{|| t|-| x|| \sim 2^{s}} \psi_{\alpha, 1}(t, x)\right| \lesssim 2^{C \delta l} 2^{-s},|x| \gtrsim 2^{l}
$$

Summing over $2^{s}>>2^{l}$ yields the bound $\lesssim 2^{C \delta l} 2^{-l}$, which better than what we need. Next, assuming $2^{s} \lesssim 2^{l}$ and arguing in the same way, we get the bound

$$
\|\left.\Gamma^{\beta}\left[\chi_{|x| \gtrsim 2^{l}} \chi_{|| t|-| x|| \sim 2^{s}} \psi_{\alpha, 1}\right]\right|_{L_{x}^{2}} \lesssim 2^{C \delta l}\left[2^{2 l} 2^{s}\right]^{\frac{1}{6}} 2^{-\frac{s}{2}} 2^{-l} \lesssim 2^{-\frac{s}{3}} 2^{C \delta l-\frac{2}{3} l}
$$

Summing over $s \geq 1$ yields the desired estimate in this range. Finally, if $|x|<<2^{l}$, apply the above reasoning to $\left\|\Gamma^{\beta}\left[\chi_{|x|<<2^{l}} \psi_{\alpha, 1}\right]\right\|_{L_{x}^{2}}$.

COROLLARY 3.2. Under the assumptions of the preceding lemma, we have the estimate

$$
\left\|\chi_{|t| \sim 2^{l}} \psi_{\alpha, 1}\right\|_{L_{t}^{2} L_{x}^{\infty}} \lesssim 2^{-\frac{l}{12}}
$$

provided $\delta>0$ is chosen small enough.

We can now prove

Proposition 3.3. Under the assumptions of Theorem 1.1 and using the preceding terminology, there exists $\left(\tilde{\psi}_{\alpha 1}, \tilde{\psi}_{\alpha 2}\right) \in \dot{H}_{x}^{\frac{1}{2}} \times \dot{H}_{x}^{-\frac{1}{2}}$ with the property

$$
\lim _{|t| \rightarrow \infty}\left\|\psi_{\alpha}(t, .)-S(t)\left(\tilde{\psi}_{\alpha 1}, \tilde{\psi}_{\alpha 2}\right)\right\|_{\dot{H}^{\frac{1}{2}}}=0, \forall \alpha
$$

REMARK 3.4. The proof actually shows that the convergence occurs at rate $|t|^{-\nu}$ for small enough $\nu>0$.

Proof. (Proposition 3.3) We decompose $\psi_{\alpha}=\psi_{\alpha, 1}+\psi_{\alpha, 2}$ for some fixed time scale $2^{l}$, viz. preceding discussion. Accordingly, denoting the right hand side of $(2.3)$ as $F(\psi)$, we write it as $F\left(\psi_{1}+\psi_{2}\right)$. Exploiting multilinearity, we write it as

$$
F(\psi)=F\left(\psi_{1}\right)+\text { error, }
$$

where error is a sum of multilinear expressions each of which contains at least one power of $\psi_{\alpha, 2}$ (for some $\alpha$ ). Then we further decompose

$$
F(\psi)=\mathbf{P}_{[-\delta l, \delta l]} F\left(\psi_{1}\right)+\left(1-\mathbf{P}_{[-\delta l, \delta l]}\right) F\left(\psi_{1}\right)+\text { error }
$$

Finally, we time localize, i. e. write

$$
F(\psi)=\sum_{l \geq 1} \phi\left(\frac{t}{2^{l}}\right) F(\psi)=\sum_{l \geq 1} \phi\left(\frac{t}{2^{l}}\right)\left[\mathbf{P}_{[-\delta l, \delta l]} F\left(\psi_{1}\right)+\left(1-\mathbf{P}_{[-\delta l, \delta l]}\right) F\left(\psi_{1}\right)+\text { error }\right]
$$

where $\psi_{1}, \psi_{2}$ in each decomposition of course depends on $l$. Now the frequency localizations implied in $\mathbf{P}_{[-\delta l, \delta l]} F\left(\psi_{1}\right)$, together with lemma 3.1 easily imply

$$
\left\|\phi\left(\frac{t}{2^{l}}\right) \mathbf{P}_{[-\delta l, \delta l]} F\left(\psi_{1}\right)\right\|_{L_{t}^{1} \dot{H}^{-\frac{1}{2}}} \lesssim 2^{-\frac{l}{12}},
$$


provided we choose $\delta>0$ small enough. Further, on account of Proposition 2.3, we obtain

$$
\left\|P_{k}\left(1-\mathbf{P}_{[-\delta l, \delta l]}\right) F\left(\psi_{1}\right)\right\|_{N[k]\left([-T, T] \times \mathbb{R}^{3}\right)}+\left\|P_{k} \operatorname{error}\right\|_{N[k]\left([-T, T] \times \mathbb{R}^{3}\right)} \lesssim e_{k}
$$

Note that error is of the form $F\left(\psi_{1}, \psi_{2}\right)$ as in Proposition 2.3. Further, due to a lemma in [24], we also have the same estimates for $P_{k} \phi\left(\frac{t}{2^{l}}\right)\left(1-\mathbf{P}_{[-\delta l, \delta l]}\right) F\left(\psi_{1}\right)$ etc. Now fix some $T_{0}>>1$ as well as $T>>T_{0}$ and write

$$
\psi_{\alpha}(t, .)=S(t)\left(\psi_{\alpha}[0]\right)+\int_{0}^{t} \eta_{T}^{+}(t-s) U(t-s) F(\psi)(s) d s
$$

where $t \in\left[T_{0}, 2 T_{0}\right]$. Here $\eta_{T}^{+}(t)$ vanishes for $t<0$, equals 1 on $[0, T]$, and is compactly supported and smooth on $(0, \infty]$. For example, upon choosing such a $\eta_{1}^{+}(t)$, one can set $\eta_{T}^{+}(t)=\eta_{1}^{+}\left(\frac{t}{T}\right)$. Then decompose

$$
\begin{aligned}
\int_{0}^{t} \eta_{T}^{+}(t-s) U(t-s) F(\psi)(s) d s= & \int_{0}^{T_{0}} \eta_{T}^{+}(t-s) U(t-s) F(\psi)(s) d s \\
& +\int_{T_{0}}^{t} \eta_{T}^{+}(t-s) U(t-s) F(\psi)(s) d s
\end{aligned}
$$

We need to show $(\mathrm{A})$ that

$$
\lim _{T_{0} \rightarrow \infty} \int_{0}^{T_{0}} \eta_{T}^{+}(t-s) U(t-s) F(\psi)(s) d s=S(t)\left(\tilde{\psi}_{\alpha 1}, \tilde{\psi}_{\alpha 2}\right)
$$

in the $\dot{H}^{\frac{1}{2}}$-sense for suitable $\left(\tilde{\psi}_{\alpha 1}, \tilde{\psi}_{\alpha 2}\right) \in \dot{H}^{\frac{1}{2}} \times \dot{H}^{-\frac{1}{2}}$, as well as (B)

$$
\lim _{T_{0} \rightarrow \infty}\left\|\int_{T_{0}}^{t} \eta_{T}^{+}(t-s) U(t-s) F(\psi)(s) d s\right\|_{\dot{H}^{\frac{1}{2}}}=0
$$

Use that $U(t-s)={\sqrt{-\triangle^{-1}}}^{-1} \sin (\sqrt{-\triangle}(t-s))={\sqrt{-\triangle^{-1}}}^{-e^{i(t-s) \sqrt{-\triangle}}-e^{-i(t-s) \sqrt{-\triangle}}}$. Hence it suffices to consider

$$
\begin{aligned}
& \int_{0}^{T_{0}} \eta_{T}^{+}(t-s) \sqrt{-\triangle}^{-1} e^{ \pm i(t-s) \sqrt{-\triangle}} F(\psi)(s) d s \\
& =\sqrt{-\triangle}^{-1} e^{ \pm i t \sqrt{-\triangle}} \int_{0}^{T_{0}} e^{\mp i s \sqrt{-\triangle}} F(\psi)(s) d s
\end{aligned}
$$

Here we use that $\eta_{T}^{+}(t-s)=1$ for $t \in\left[T_{0}, 2 T_{0}\right], 0 \leq s \leq t$, since $T>>T_{0}$. We will show that the limit

$$
\lim _{T_{0} \rightarrow \infty} \int_{0}^{T_{0}} e^{\mp i s \sqrt{-\triangle}} F(\psi)(s) d s
$$

exists in $\dot{H}^{-\frac{1}{2}}$, from which (A) follows. Consider

$$
\int_{T_{0}}^{T_{0}^{\prime}} e^{\mp i s \sqrt{-\triangle}} F(\psi)(s) d s=\sum_{2^{l} \gtrsim T_{0}} \int_{T_{0}}^{T_{0}^{\prime}} \phi\left(\frac{s}{2^{l}}\right) e^{\mp i s \sqrt{-\triangle}} F(\psi)(s) d s
$$

Then note that

$$
\left\|\int_{T_{0}}^{T_{0}^{\prime}} \phi\left(\frac{s}{2^{l}}\right) e^{\mp i s \sqrt{-\triangle}} F(\psi)(s) d s\right\|_{\dot{H}^{-\frac{1}{2}}}=\sup _{\|g\|_{\dot{H}^{\frac{1}{2}}}=1}\left\langle\int_{T_{0}}^{T_{0}^{\prime}} \phi\left(\frac{s}{2^{l}}\right) e^{\mp i s \sqrt{-\triangle}} F(\psi)(s) d s, g\right\rangle
$$


Now, using (3.1), we get

$$
\left|\left\langle\int_{T_{0}}^{T_{0}^{\prime}} \phi\left(\frac{s}{2^{l}}\right) e^{\mp i s \sqrt{-\triangle}} \mathbf{P}_{[-\delta l, \delta l]} F\left(\psi_{1}\right)(s) d s, g\right\rangle\right| \lesssim 2^{-\frac{l}{12}} \lesssim T_{0}^{-\frac{1}{12}}
$$

Further, write

$$
\begin{aligned}
& \left\langle\int_{T_{0}}^{T_{0}^{\prime}} \phi\left(\frac{s}{2^{l}}\right) e^{\mp i s \sqrt{-\triangle}}\left(1-\mathbf{P}_{[-\delta l, \delta l]}\right) F\left(\psi_{1}\right)(s) d s, g\right\rangle \\
& =\sum_{k} \int_{-\infty}^{\infty} \int_{\mathbb{R}^{3}} \phi\left(\frac{s}{2^{l}}\right) P_{k}\left(1-\mathbf{P}_{[-\delta l, \delta l]}\right) F\left(\psi_{1}\right)(s, x) e^{ \pm i s \sqrt{-\triangle}} \tilde{P}_{k} g(x) d x d s
\end{aligned}
$$

Here $\tilde{P}_{k}$ is a multiplier like $P_{k}$ but satisfying $\tilde{P}_{k} P_{k}=P_{k}$. Then use that [24] $\left\|\phi\left(\frac{s}{2^{l}}\right) e^{ \pm i s \sqrt{-\triangle}} \tilde{P}_{k} g\right\|_{S[k]} \lesssim\|g\|_{\dot{H}^{\frac{1}{2}}}$, as well as $\mid\left\langle P_{k} F, \psi>\right| \lesssim\|F\|_{N[k]}\|\psi\|_{S[k]}$. Using Cauchy-Schwarz, we conclude that

$$
\begin{aligned}
& \left|\left\langle\int_{T_{0}}^{T_{0}^{\prime}} \phi\left(\frac{s}{2^{l}}\right) e^{\mp i s \sqrt{-\triangle}}\left(1-\mathbf{P}_{[-\delta l, \delta l]}\right) F\left(\psi_{1}\right)(s) d s, g\right\rangle\right| \\
& \lesssim \sum_{k \in \mathbb{Z}}\left\|P_{k} \phi\left(\frac{s}{2^{l}}\right)\left(1-\mathbf{P}_{[-\delta l, \delta l]}\right) F\left(\psi_{1}\right)(s)\right\|_{N[k]}\left\|\tilde{P}_{k} g\right\|_{\dot{H}^{\frac{1}{2}}} \lesssim\left(\sum_{k \in \mathbb{Z}} e_{k}^{2}\right)^{\frac{1}{2}} \lesssim 2^{-\nu l} \lesssim T_{0}^{-\nu}
\end{aligned}
$$

for suitable $\nu>0$. Of course the same argument applies to the contribution of error in (3.1), whence summing over $l$ with $2^{l} \sim T_{0}$ and letting $T_{0} \rightarrow \infty$, we obtain (A), i. e. the $\dot{H}^{\frac{1}{2}}$ convergence of $\int_{0}^{T_{0}} e^{\mp i s \sqrt{-\triangle}} F(\psi)(s) d s$. The argument for establishing (B), i. e.

$$
\lim _{T_{0} \rightarrow \infty}\left\|\int_{T_{0}}^{t} \eta_{T}^{+}(t-s) U(t-s) F(\psi)(s) d s\right\|_{\dot{H}^{\frac{1}{2}}}=0,
$$

is no different, using Theorem 4.4 as well as the fact (see e. g. [24]) that

$$
\left\|\int_{T_{0}}^{t} \eta_{T}^{+}(t-s) U(t-s) F(\psi)(s) d s\right\|_{S[k]\left([-T, T] \times \mathbb{R}^{3}\right)} \lesssim\|F(\psi)\|_{N[k]\left([-T, T] \times \mathbb{R}^{3}\right)},
$$

and applying the same reasoning to the term "error", the proof of Proposition 3.3 is complete.

We now show how to deduce scattering of the original derivative components from Proposition 3.3. We have

Proposition 3.5. Under the assumptions of the preceding Proposition, we have

$$
\lim _{|t| \rightarrow \infty}\left\|\phi_{\alpha}-S(t)\left(\tilde{\psi}_{\alpha 1}, \tilde{\psi}_{\alpha 2}\right)\right\|_{\dot{H}^{\frac{1}{2}}}=0
$$

Furthermore, there exist pairs $\left(f_{1}, g_{1}\right) \in \dot{H}^{\frac{3}{2}} \times \dot{H}^{\frac{1}{2}},\left(f_{2}, g_{2}\right) \in \dot{H}^{\frac{3}{2}} \times \dot{H}^{\frac{1}{2}}$, such that

$$
\lim _{|t| \rightarrow \infty}\left\|\mathbf{x}(t, .)-S(t)\left(f_{1}, g_{1}\right)\right\|_{\dot{H}^{\frac{3}{2}}}=0, \lim _{|t| \rightarrow \infty}\left\|\mathbf{y}(t, .)-S(t)\left(f_{2}, g_{2}\right)\right\|_{\dot{H}^{\frac{3}{2}}}=0
$$

Proof. (Proposition 3.5). Recall that $\phi_{\alpha}=\psi_{\alpha} e^{i \triangle^{-1} \sum_{j=1}^{3} \partial_{j} \phi_{j}^{1}}$. We shall need the following

Lemma 3.6. We have

$$
\left\|P_{k} e^{i \triangle^{-1} \sum_{j=1}^{3} \partial_{j} \phi_{j}^{1}}\right\|_{\dot{H}^{\frac{3}{2}}}+\left\|P_{k} \phi_{\alpha}\right\|_{\dot{H}^{\frac{1}{2}}} \lesssim d_{k}
$$


Proof. (Lemma 3.6) Expand

$$
\begin{aligned}
& P_{k}\left[e^{i \triangle^{-1} \sum_{j=1}^{3} \partial_{j} \phi_{j}^{1}} \|_{\dot{H}^{\frac{3}{2}}}\right] \\
& =P_{k} \sum_{l=1}^{3} \partial_{l} \triangle^{-1}\left[P_{<k-5}\left(\sum_{j=1}^{3} \triangle^{-1} \partial_{l} \partial_{j} \phi_{j}^{1}\right) P_{[k-2, k+2]} e^{i \triangle^{-1} \sum_{j=1}^{3} \partial_{j} \phi_{j}^{1}} \|_{\dot{H}^{\frac{3}{2}}}\right. \\
& +P_{k} \sum_{l=1}^{3} \partial_{l} \triangle^{-1}\left[P_{[k-5, k+5]}\left(\sum_{j=1}^{3} \triangle^{-1} \partial_{l} \partial_{j} \phi_{j}^{1}\right) P_{<k+10} e^{i \triangle^{-1} \sum_{j=1}^{3} \partial_{j} \phi_{j}^{1}} \|_{\dot{H}^{\frac{3}{2}}}\right] \\
& +P_{k} \sum_{l=1}^{3} \partial_{l} \triangle^{-1}\left[P_{>k+5}\left(\sum_{j=1}^{3} \triangle^{-1} \partial_{l} \partial_{j} \phi_{j}^{1}\right) P_{>k+2} e^{i \triangle^{-1} \sum_{j=1}^{3} \partial_{j} \phi_{j}^{1}} \|_{\dot{H}^{\frac{3}{2}}}\right]
\end{aligned}
$$

Similarly, we have the identity

$$
\begin{aligned}
P_{k} \phi_{\alpha}= & P_{k}\left[P_{<k-5} \psi_{\alpha} P_{[k-2, k+2]} e^{\left.i \triangle^{-1} \sum_{j=1}^{3} \partial_{j} \phi_{j}^{1}\right]}\right. \\
& +P_{k}\left[P_{[k-5, k+5]} \psi_{\alpha} P_{<k+10} e^{i \triangle^{-1} \sum_{j=1}^{3} \partial_{j} \phi_{j}^{1}}\right] \\
& +P_{k}\left[P_{>k+5} \psi_{\alpha} P_{>k+2} e^{i \triangle^{-1} \sum_{j=1}^{3} \partial_{j} \phi_{j}^{1}}\right]
\end{aligned}
$$

We infer from the first identity above that

$$
\begin{aligned}
\| P_{k}\left[e^{i \triangle^{-1} \sum_{j=1}^{3} \partial_{j} \phi_{j}^{1}} \|_{\dot{H}^{\frac{3}{2}}} \lesssim\right. & \left\|P_{[k-2, k+2]} e^{i \triangle^{-1} \sum_{j=1}^{3} \partial_{j} \phi_{j}^{1}}\right\|_{\dot{H}^{\frac{3}{2}}} \max _{j}\left\{\left\|\phi_{j}^{1}\right\|_{\dot{H}^{\frac{1}{2}}}\right\} \\
& +\left\|P_{k-5, k+5} \phi_{j}^{1}\right\|_{\dot{H}^{\frac{1}{2}}}+\sum_{l>k+5} 2^{\frac{k-l}{2}}\left\|P_{l} \phi_{j}^{1}\right\|_{\dot{H}^{\frac{1}{2}}}
\end{aligned}
$$

Further, from the 2nd equality above we infer

$$
\begin{aligned}
\left\|P_{k} \phi_{\alpha}\right\|_{\dot{H}^{\frac{1}{2}}} \lesssim & \left\|P_{[k-2, k+2]} e^{i \triangle^{-1} \sum_{j=1}^{3} \partial_{j} \phi_{j}^{1}}\right\|_{\dot{H}^{\frac{3}{2}}}\left\|P_{<k-5} \psi_{\alpha}\right\|_{\dot{H}^{\frac{1}{2}}} \\
& +\left\|P_{[k-5, k+5]} \psi_{\alpha}\right\|_{\dot{H}^{\frac{1}{2}}}+\sum_{l>k+5} 2^{\frac{k-l}{2}}\left\|P_{l} \psi_{\alpha}\right\|_{\dot{H}^{\frac{1}{2}}}
\end{aligned}
$$

Substituting the latter inequality into the former, we obtain

$$
\begin{aligned}
\|\left. P_{k}\left[e^{\left.i \triangle^{-1} \sum_{j=1}^{3} \partial_{j} \phi_{j}^{1}\right]}\right]\right|_{\dot{H}^{\frac{3}{2}}} \lesssim & \left\|P_{[k-2, k+2]} e^{i \triangle^{-1} \sum_{j=1}^{3} \partial_{j} \phi_{j}^{1}}\right\|_{\dot{H}^{\frac{3}{2}}} \max _{j}\left\{\left\|\phi_{j}^{1}\right\|_{\dot{H}^{\frac{1}{2}}}\right\} \\
& +\left\|P_{[k-7, k+7]} e^{i \triangle^{-1} \sum_{j=1}^{3} \partial_{j} \phi_{j}^{1}}\right\|_{\dot{H}^{\frac{3}{2}}}\left\|P_{<k} \psi_{\alpha}\right\|_{\dot{H}^{\frac{1}{2}}} \\
& +\left\|P_{[k-10, k+10]} \psi_{\alpha}\right\|_{\dot{H}^{\frac{1}{2}}}+\sum_{l>k} 2^{\frac{k-l}{2}}\left\|P_{l} \psi_{\alpha}\right\|_{\dot{H}^{\frac{1}{2}}} \\
& +\sum_{l>k} 2^{\frac{k-l}{2}}\left\|P_{l} \psi_{j}\right\|_{\dot{H}^{\frac{1}{2}}}
\end{aligned}
$$

Using the definition of $d_{k}$, this implies

$$
\left.\left\|P_{k}\left[e^{i \triangle^{-1} \sum_{j=1}^{3} \partial_{j} \phi_{j}^{1}}\right]\right\|\right|_{\dot{H}^{\frac{3}{2}}} \lesssim \epsilon \|\left. P_{[k-7, k+7]}\left[e^{i \triangle^{-1} \sum_{j=1}^{3} \partial_{j} \phi_{j}^{1}}\right]\right|_{\dot{H}^{\frac{3}{2}}}+d_{k}
$$

Iterating and choosing $\epsilon$ small enough, we obtain the desired bound

$$
\left\|P_{k}\left[e^{i \triangle^{-1} \sum_{j=1}^{3} \partial_{j} \phi_{j}^{1}}\right]\right\|_{\dot{H}^{\frac{3}{2}}} \lesssim d_{k},
$$

which in conjunction with the above easily implies $\left\|P_{k} \phi_{\alpha}\right\|_{L_{x}^{2}} \lesssim d_{k}$. 
Returning to the proof of the Proposition, fix a time scale $|t| \sim 2^{l}, l>>1$, and correspondingly decompose $\psi_{\alpha}=\psi_{\alpha, 1}+\psi_{\alpha, 2}$. Then we can write $\phi_{\alpha}=\phi_{\alpha, 1}+\phi_{\alpha, 2}$ with

$$
\phi_{\alpha, 1}=\psi_{\alpha, 1} e^{i \triangle^{-1} \sum_{j=1}^{3} \partial_{j} \phi_{j}^{1}}, \phi_{\alpha, 2}=\psi_{\alpha, 2} e^{i \triangle^{-1} \sum_{j=1}^{3} \partial_{j} \phi_{j}^{1}}
$$

Using reasoning as above, it is then clear that

$$
\left\|\phi_{\alpha, 1}\right\|_{L_{x}^{\infty}} \lesssim 2^{-\frac{7}{12} l},\left\|\phi_{\alpha, 2}\right\|_{\dot{H}^{\frac{1}{2}}} \lesssim 2^{-\nu l},
$$

for some $\nu>0$. Indeed, we can strengthen the latter to $\left\|\phi_{\alpha, 2}\right\|_{\dot{B}^{\frac{1}{2}, 1}} \lesssim 2^{-\nu l}$ and similarly $\left\|P_{[-\nu l, \nu l]^{c}} \phi_{\alpha, 1}\right\|_{\dot{B}^{\frac{1}{2}, 1}} \lesssim 2^{-\nu^{\prime} l}$ for some $\nu^{\prime}>0$. By splitting

$$
\triangle^{-1} \sum_{j=1}^{3} \partial_{j} \phi_{j}^{1}=\triangle^{-1} \sum_{j=1}^{3} P_{[-\nu l, \nu l]} \partial_{j} \phi_{j}^{1}+\triangle^{-1} \sum_{j=1}^{3} P_{[-\nu l, \nu l]^{c}} \partial_{j} \phi_{j}^{1},
$$

and using the decomposition $\phi_{j}^{1}=\phi_{j 1}^{1}+\phi_{j 2}^{1}$ from above, we then deduce

$$
\left\|\triangle^{-1} \sum_{j=1}^{3} \partial_{j} \phi_{j}^{1}(t, .)\right\|_{L_{x}^{\infty}} \lesssim 2^{-\nu^{\prime} l},|t| \sim 2^{l}
$$

for some $\nu^{\prime}>0$. Now write

$$
\phi_{\alpha}-S(t)\left(\tilde{\psi}_{\alpha 1}, \tilde{\psi}_{\alpha 2}\right)=\left(e^{i \triangle^{-1} \sum_{j=1}^{3} \partial_{j} \phi_{j}^{1}}-1\right) \psi_{\alpha}+\psi_{\alpha}-S(t)\left(\tilde{\psi}_{\alpha 1}, \tilde{\psi}_{\alpha 2}\right)
$$

We need to show that the first expression on the right converges to zero as $t \rightarrow \infty$, with respect to $\dot{H}^{\frac{1}{2}}$. Once again decompose

$$
\begin{aligned}
P_{k}\left[\left(e^{i \triangle^{-1} \sum_{j=1}^{3} \partial_{j} \phi_{j}^{1}}-1\right) \psi_{\alpha}\right]= & P_{k}\left[P_{[k-10, k+10]}\left(e^{i \triangle^{-1} \sum_{j=1}^{3} \partial_{j} \phi_{j}^{1}}-1\right) P_{<k-5} \psi_{\alpha}\right] \\
& +P_{k}\left[P_{<k+10}\left(e^{i \triangle^{-1} \sum_{j=1}^{3} \partial_{j} \phi_{j}^{1}}-1\right) P_{[k-5, k+5]} \psi_{\alpha}\right] \\
& +P_{k}\left[P_{>k+2}\left(e^{i \triangle^{-1} \sum_{j=1}^{3} \partial_{j} \phi_{j}^{1}}-1\right) P_{>k+5} \psi_{\alpha}\right]
\end{aligned}
$$

For the first term on the right, we can bound

$$
\begin{aligned}
& \left\|P_{k}\left[P_{[k-10, k+10]}\left(e^{i \triangle^{-1} \sum_{j=1}^{3} \partial_{j} \phi_{j}^{1}}-1\right) P_{<k-5} \psi_{\alpha}\right]\right\|_{\dot{H}^{\frac{1}{2}}} \\
& \lesssim\left\|P_{[k-10, k+10]}\left(e^{i \triangle^{-1} \sum_{j=1}^{3} \partial_{j} \phi_{j}^{1}}-1\right)\right\|_{\dot{H}^{\frac{3}{2}}}\left\|P_{<k-5} \psi_{\alpha}\right\|_{\dot{H}^{\frac{1}{2}}}
\end{aligned}
$$

Thus, using Lemma 3.6, if we restrict to $|k|>\nu l$, we obtain exponential decay in $l$. Hence we can restrict to $|k|<\nu l$. Then use the splitting $\psi_{\alpha}=\psi_{\alpha, 1}+\psi_{\alpha, 2}$ and estimate

$$
\begin{aligned}
& \left\|P_{k}\left[P_{[k-10, k+10]}\left(e^{i \triangle^{-1} \sum_{j=1}^{3} \partial_{j} \phi_{j}^{1}}-1\right) P_{<k-5} \psi_{\alpha, 1}\right]\right\|_{\dot{H}^{\frac{1}{2}}} \\
& \lesssim\left\|P_{[k-10, k+10]}\left(e^{i \triangle^{-1} \sum_{j=1}^{3} \partial_{j} \phi_{j}^{1}}-1\right)\right\|_{\dot{H}^{\frac{3}{2}}} 2^{-k}\left\|\psi_{\alpha, 1}\right\|_{L_{x}^{\infty}} \lesssim 2^{\nu l-\frac{7}{12} l} 2^{-k} d_{k}
\end{aligned}
$$

Choosing $\nu>0$ small enough and summing over $|k|<\nu l$ still results in exponential decay in $l$. Further, we have

$$
\begin{aligned}
& \left\|P_{k}\left[P_{[k-10, k+10]}\left(e^{i \triangle^{-1} \sum_{j=1}^{3} \partial_{j} \phi_{j}^{1}}-1\right) P_{<k-5} \psi_{\alpha, 2}\right]\right\|_{\dot{H}^{\frac{1}{2}}} \\
& \lesssim\left\|\left.P_{[k-10, k+10]}\left(e^{i \triangle^{-1} \sum_{j=1}^{3} \partial_{j} \phi_{j}^{1}}-1\right)\right|_{\dot{H}^{\frac{3}{2}}} 2^{-k}|| P_{<k-5} \psi_{\alpha, 2}\right\|_{L_{x}^{\infty}} \lesssim e_{k},
\end{aligned}
$$

where in the last step we have used Bernstein's inequality and the Sobolev embedding as well as the definition of $e_{k}$. Square-summing over $k$ results in an exponential gain in $l$. 
Next, consider the term $P_{k}\left[P_{<k+10}\left(e^{i \triangle^{-1} \sum_{j=1}^{3} \partial_{j} \phi_{j}^{1}}-1\right) P_{[k-5, k+5]} \psi_{\alpha}\right]$. Here we obtain exponential decay from

$$
\left\|\left(e^{i \triangle^{-1} \sum_{j=1}^{3} \partial_{j} \phi_{j}^{1}(t, .)}-1\right)\right\|_{L_{x}^{\infty}} \lesssim 2^{-\nu^{\prime} l},|t| \sim 2^{l},
$$

which follows from the bound on the exponent obtained further above. The term $P_{k}\left[P_{>k+2}\left(e^{i \triangle^{-1} \sum_{j=1}^{3} \partial_{j} \phi_{j}^{1}}-1\right) P_{>k+5} \psi_{\alpha}\right]$ is handled similarly.

Finally, we also need to demonstrate scattering for the original coordinate functions $\mathbf{x}, \mathbf{y}$. Recall that we have

$$
\log \left[\frac{\mathbf{y}}{\mathbf{y}_{\infty}}\right]=\triangle^{-1} \sum_{j=1}^{3} \partial_{j} \phi_{j}^{2}
$$

Reasoning as above for $\triangle^{-1} \sum_{j=1}^{3} \partial_{j} \phi_{j}^{1}$, we obtain

$$
\lim _{|t| \rightarrow \infty}\left\|\triangle^{-1} \sum_{j=1}^{3} \partial_{j} \phi_{j}^{2}(t, .)\right\|_{L_{x}^{\infty}}=0
$$

with a small polynomial decay rate in $t$. The already proved fact that $\phi_{j}(t,)=$. $S(t)\left(\tilde{\psi}_{\alpha 1}, \tilde{\psi}_{\alpha 2}\right)+o_{\dot{H} \frac{1}{2}}(1)$ yields

$$
\log \left[\frac{\mathbf{y}}{\mathbf{y}_{\infty}}\right](t, .)=S(t)\left(\triangle^{-1} \sum_{j=1}^{3} \partial_{j} \tilde{\psi}_{j 1}^{2}, \triangle^{-1} \sum_{j=1}^{3} \partial_{j} \tilde{\psi}_{j 2}^{2}\right)+o_{\dot{H}^{\frac{3}{2}}}(1)
$$

Here we of course write $\tilde{\psi}_{\alpha 1,2}=\tilde{\psi}_{\alpha 1,2}^{1}+i \tilde{\psi}_{\alpha 1,2}^{2}$. Further, invoking (3.2) and reasoning as above, we obtain from here

$$
\frac{\mathbf{y}}{\mathbf{y}_{\infty}}(t, .)=S(t)\left(\triangle^{-1} \sum_{j=1}^{3} \partial_{j} \tilde{\psi}_{j 1}^{2}, \triangle^{-1} \sum_{j=1}^{3} \partial_{j} \tilde{\psi}_{j 2}^{2}\right)+o_{\dot{H}^{\frac{3}{2}}}(1)
$$

From the relation $\phi_{j}^{1}=\frac{\partial_{j} \mathbf{x}}{\mathbf{y}}$, we obtain in the same fashion that

$$
\frac{\mathbf{x}}{\mathbf{y}_{\infty}}=S(t)\left(\triangle^{-1} \sum_{j=1}^{3} \partial_{j} \tilde{\psi}_{j 1}^{1}, \triangle^{-1} \sum_{j=1}^{3} \partial_{j} \tilde{\psi}_{j 2}^{1}\right)+o_{\dot{H}^{\frac{3}{2}}}(1)
$$

We further have the following

COROLlary 3.7. (of preceding proof) We have the bound

$$
\left\|\left((\mathbf{x}, \mathbf{y})-\left(\mathbf{x}_{\infty}, \mathbf{y}_{\infty}\right)\right)(t, .)\right\|_{L_{x}^{\infty}} \lesssim|t|^{-\nu}
$$

for $\nu>0$ sufficiently small and large $|t|$. Here $\left.\left(\mathbf{x}_{\infty}, \mathbf{y}_{\infty}\right)\right)$ are the values of $u(0, x)$ for large $|x|$, i. e. the "data at infinity".

\section{The core propositions}

We now outline the proofs of Proposition 2.1, Proposition 2.2, as well as Proposition 2.3. We observe that these are essentially contained in $[\mathbf{9}],[\mathbf{1 0}]$, the only new ingredient being the presence of the vector fields $\Gamma^{\beta}$. We shall refer some details to these papers. We begin by collecting some 
4.1. Basic facts concerning the spaces $S[k], N[k]$. .

LEMMA 4.1. ([9]) We have

$$
\left\|R_{\nu} P_{k} \psi\right\|_{L_{t}^{p} L_{x}^{q}} \lesssim 2^{\left[\left(\frac{1}{4}-\frac{1}{p}\right)+\left(\frac{3}{4}-\frac{3}{p}\right)\right] k}\left\|P_{k} \psi\right\|_{S[k]}, \nu=0,1,2,3
$$

provided $\frac{1}{p}+\frac{1}{q}<\frac{1}{2}$, with implicit constant possibly depending on $p, q$. Also, we have

$$
\left\|P_{k} \psi\right\|_{S[k]} \lesssim\left\|P_{k} \nabla_{x, t} \psi\right\|_{\dot{X}_{k}^{-\frac{1}{2}, \frac{1}{2}, 1}}
$$

The first part of this lemma allows us to control some Strichartz type norms. This shall be especially handy when estimating terms of high degree of multilinearity.

Lemma 4.2. (e. g. [9]) The following estimate holds for suitable $\delta>0$ :

$$
\left\|P_{k} Q_{j}\left[P_{k_{1}} \psi_{1} P_{k_{2}} \psi_{2}\right]\right\|_{\dot{X}^{-\frac{1}{2}, \frac{1}{2}, \infty}} \lesssim 2^{\delta \min \left\{j-\min \left\{k, k_{1}, k_{2}\right\}, 0\right\}} 2^{-\left|k_{1}-k_{2}\right|} \prod_{j=1,2}\left\|P_{k_{j}} \psi_{j}\right\|_{S\left[k_{j}\right]}
$$

Furthermore, for any $\mu>0$ we have

$$
\left\|P_{k}\left[P_{k_{1}} \psi_{1} P_{k_{2}} \psi_{2}\right]\right\|_{L_{t}^{2} L_{x}^{2+\mu}} \lesssim 2^{\frac{\mu}{4 \mu+2} k} 2^{-\frac{\left|k_{1}-k_{2}\right|}{2}} \prod_{j=1,2}\left\|P_{k_{j}} \psi_{j}\right\|_{S\left[k_{j}\right]}
$$

Finally, the following bound obtains ${ }^{4}$ for $p>2$ :

$$
\begin{aligned}
& \left\|P_{k}\left[R_{\nu} P_{k_{1}} \psi_{1} R_{j} P_{k_{2}} \psi_{2}-R_{j} P_{k_{1}} \psi_{1} R_{\nu} P_{k_{2}} \psi_{2}\right]\right\|_{L_{t}^{2} L_{x}^{p}} \\
& \lesssim 2^{\left(\frac{3}{2}-\frac{3}{p}\right) k} 2^{\frac{\min \left\{k, k_{1,2}\right\}-\max \left\{k, k_{1,2}\right\}}{2}} \prod_{i=1,2}\left\|P_{k_{i}} \psi_{i}\right\|_{S\left[k_{i}\right]}
\end{aligned}
$$

Proof. (Lemma 4.2) Only the last part requires a justification, and this only in the case $k_{1}>>k$. We decompose

$$
\begin{aligned}
& P_{k}\left[R_{\nu} P_{k_{1}} \psi_{1} R_{j} P_{k_{2}} \psi_{2}-R_{j} P_{k_{1}} \psi_{1} R_{\nu} P_{k_{2}} \psi_{2}\right] \\
& =P_{k} Q_{<k+20}\left[R_{\nu} P_{k_{1}} \psi_{1} R_{j} P_{k_{2}} \psi_{2}-R_{j} P_{k_{1}} \psi_{1} R_{\nu} P_{k_{2}} \psi_{2}\right] \\
& +P_{k} Q_{\geq k+20}\left[R_{\nu} P_{k_{1}} \psi_{1} R_{j} P_{k_{2}} \psi_{2}-R_{j} P_{k_{1}} \psi_{1} R_{\nu} P_{k_{2}} \psi_{2}\right]
\end{aligned}
$$

For the first term, use a simple algebraic identity and estimate

$$
\begin{aligned}
& \left\|P_{k} Q_{<k+20}\left[R_{\nu} P_{k_{1}} \psi_{1} R_{j} P_{k_{2}} \psi_{2}-R_{j} P_{k_{1}} \psi_{1} R_{\nu} P_{k_{2}} \psi_{2}\right]\right\|_{L_{t}^{2} L_{x}^{p}} \\
& \quad \leq \| P_{k} Q_{<k+20} \partial_{\nu}\left[\nabla ^ { - 1 } P _ { k _ { 1 } } \psi _ { 1 } R _ { j } P _ { k _ { 2 } } \psi _ { 2 } \| _ { L _ { t } ^ { 2 } L _ { x } ^ { p } } + \| P _ { k } Q _ { < k + 2 0 } \partial _ { j } \left[R_{\nu} P_{k_{1}} \psi_{1} \nabla^{-1} P_{k_{2}} \psi_{2} \|_{L_{t}^{2} L_{x}^{p}}\right.\right. \\
& \lesssim 2^{-k_{1}}\left\|P_{k_{1}} \psi_{1}\right\|_{L_{t}^{4} L_{x}^{2 p}}\left\|P_{k_{2}} \psi_{2}\right\|_{L_{t}^{4} L_{x}^{2 p}}
\end{aligned}
$$

and from here the claimed inequality follows easily. Further, we decompose

$$
\begin{aligned}
& P_{k} Q_{\geq k+20}\left[R_{\nu} P_{k_{1}} \psi_{1} R_{j} P_{k_{2}} \psi_{2}-R_{j} P_{k_{1}} \psi_{1} R_{\nu} P_{k_{2}} \psi_{2}\right] \\
& =P_{k} Q_{\geq k+20}\left[R_{\nu} P_{k_{1}} Q_{\geq k+10} \psi_{1} R_{j} P_{k_{2}} \psi_{2}-R_{j} P_{k_{1}} Q_{\geq k+10} \psi_{1} R_{\nu} P_{k_{2}} \psi_{2}\right] \\
& +P_{k} Q_{\geq k+20}\left[R_{\nu} P_{k_{1}} Q_{<k+10} \psi_{1} R_{j} P_{k_{2}} Q_{\geq k+10} \psi_{2}-R_{j} P_{k_{1}} Q_{<k+10} \psi_{1} R_{\nu} P_{k_{2}} Q_{\geq k+10} \psi_{2}\right] \\
& +P_{k} Q_{\geq k+20}\left[R_{\nu} P_{k_{1}} Q_{<k+10} \psi_{1} R_{j} P_{k_{2}} Q_{<k+10} \psi_{2}-R_{j} P_{k_{1}} Q_{<k+10} \psi_{1} R_{\nu} P_{k_{2}} Q_{<k+10} \psi_{2}\right]
\end{aligned}
$$

\footnotetext{
${ }^{4}$ One can significantly strengthen this estimate and also include the case $p=2$, see e. $\mathrm{g}$. [11], but we don't need this here.
} 
The first two terms are estimated similarly: for example, we have

$$
\begin{aligned}
& \left\|P_{k} Q_{\geq k+20}\left[R_{\nu} P_{k_{1}} Q_{\geq k+10} \psi_{1} R_{j} P_{k_{2}} \psi_{2}-R_{j} P_{k_{1}} Q_{\geq k+10} \psi_{1} R_{\nu} P_{k_{2}} \psi_{2}\right]\right\|_{L_{t}^{2} L_{x}^{2}} \\
& \lesssim 2^{\frac{3 k}{2}}\left\|\nabla_{x, t} \nabla^{-1} P_{k_{1}} Q_{\geq k+10} \psi_{1}\right\|_{L_{t}^{2} L_{x}^{2}}\left\|\nabla_{x, t} \nabla^{-1} P_{k_{1}} Q_{\geq k+10} \psi_{1}\right\|_{L_{t}^{\infty} L_{x}^{2}} \\
& \lesssim 2^{\frac{3 k}{2}-\frac{k}{2}-k_{1}} \prod_{i=1,2}\left\|P_{k_{i}} \psi_{i}\right\|_{S\left[k_{i}\right]},
\end{aligned}
$$

and the inequality of the Lemma follows from Bernstein's inequality. Finally, for the last term above, upon freezing the output to modulation $2^{l}, l \geq k+10$ (i. e. apply an operator $Q_{l}$ to the expression), use that we may assume $k_{1}=l+O(1)$. Then the inequality follows from the same calculation as at the end the of the proof of Lemma 4.8 below, after summing over $l$.

Of fundamental importance is furthermore the following

Lemma 4.3. ([24], [10])Let $j \leq \min \left\{k_{1}, k_{2}\right\}+O(1)$. Also, let $F$ and $\psi$ be Schwartz functions, the former at frequency $\sim 2^{k_{1}}$ and modulation (distance of the space-time Fourier support to the light cone) $\sim 2^{j}$, the latter at frequency $2^{k_{2}}$. Then the following inequalities hold for suitably small $\delta_{1,2}>0$ :

$$
\begin{gathered}
\left\|\left.\left.P_{k}(F \psi)\right|_{N[k]} \lesssim 2^{-\delta_{1} \mid k-\max \left\{k_{1}, k_{2}\right\}}\right|^{-\delta_{2}\left|j-\min \left\{k_{1}, k_{2}\right\}\right|}\right\| F\left\|_{\dot{X}_{k_{1}}^{\frac{1}{2},-\frac{1}{2}, \infty}}\right\| \psi \|_{S\left[k_{2}\right]} \\
\left\|\nabla_{x} P_{k}(F \psi)\right\|_{N[k]} \lesssim 2^{-\delta_{1}\left|k-\max \left\{k_{1}, k_{2}\right\}\right|} 2^{-\delta_{2}\left|j-\min \left\{k_{1}, k_{2}\right\}\right|}\left\|\left.F\right|_{\dot{X}_{k_{1}}^{\frac{1}{2},-\frac{1}{2}, \infty}}\right\| \nabla_{x} \psi\|\|_{S\left[k_{2}\right]}
\end{gathered}
$$

Finally, the relation between $S[k]$ ad $N[k]$ is obtained via the following

THEOREM 4.4. The following inequality holds:

$$
\left\|P_{k} \phi\right\|_{S[k]\left([-T, T] \times \mathbb{R}^{3}\right)} \lesssim\left\|\square P_{k} \phi\right\|_{N[k]\left([-T, T] \times \mathbb{R}^{3}\right)}+\left.\|\phi[0]\|\right|_{\dot{H}^{\frac{1}{2}} \times \dot{H}^{-\frac{1}{2}}}
$$

The proof of this follows from simple modifications of the one given in [24]. We now give the proof of Propositon 2.1

Proof. (Proposition 2.1). We fix a number $l>>1$, and construct a solution $\psi_{\alpha, 1}$ (for all $\alpha$ ) on the time interval $\left[-2^{l+2}, 2^{l+2}\right]$. This solution is obtained via simple Banach iteration: specifically, we require that the iterates $\psi_{\alpha, 1, j}, j \geq 1$, all be smooth functions which are supported on a compact time interval $\left[-T^{\prime}, T^{\prime}\right]$, $T^{\prime}>>2^{l}$, and furthermore solve $(2.6)$ on the interval $\left[-2^{l+2}, 2^{l+2}\right]$. The iterative step is given by the following:

$$
\psi_{\alpha, 1, j+1}(t, .):=\eta_{T}(t) S(t)\left(\psi_{\alpha}[0]\right)+\int_{0}^{t} \eta_{T}(t-s) \mathbf{P}_{[-\delta l, \delta l]} F\left(\psi_{1, j}\right)(s) d s, j \geq 1
$$

where $T^{\prime}>>T>>2^{l}$. Furthermore, we start the iteration with

$$
\psi_{\alpha, 1,1}(t, .):=\eta_{T}(t) S(t)\left(\psi_{\alpha}[0]\right)
$$

Here $\eta_{T}(t)$ equals 1 on $[-T, T]$ and smoothly truncates to a dilate of this interval, contained within $\left[-T^{\prime}, T^{\prime}\right]$. Proposition 2.1 now follows from theorem 4.4 and the following

Proposition 4.5. Assume we have the bounds

$$
\left\|P_{k} \psi_{\alpha, 1, j}\right\|_{S[k]\left([-T, T] \times \mathbb{R}^{3}\right)} \leq M d_{k}, \forall k \in \mathbb{Z}
$$


Further, assume we have

$$
\begin{gathered}
\sum_{0 \leq|\beta| \leq 2}\left\|P_{k} \Gamma^{\beta} \psi_{\alpha, 1, j}\right\|_{S[k]\left([-T, T] \times \mathbb{R}^{3}\right)} \leq \tilde{M} 2^{C \delta l} c_{k}, \forall k \in \mathbb{Z} \\
\left\|P_{k} \nabla_{x}^{N} \psi_{\alpha, 1, j}\right\|_{S[k]\left([-T, T] \times \mathbb{R}^{3}\right)} \leq M_{N} d_{k}, \forall k \in \mathbb{Z}
\end{gathered}
$$

Here the numbers $c_{k}, d_{k}$ are as in Proposition 2.1, and $N \geq 1$ is arbitrary. Then, provided $M, \tilde{M}$ are large enough absolute constants and $\epsilon>0$ is small enough (again with $\epsilon$ as in Proposition 2.1), and $M_{N}, M_{N-1} \ldots$ are large enough constants depending on the initial data (and $N$ ), we infer the bounds

$$
\left\|P_{k} \psi_{\alpha, 1, j+1}\right\|_{S[k]\left([-T, T] \times \mathbb{R}^{3}\right)} \leq[\epsilon M+C] d_{k}, \forall k \in \mathbb{Z}
$$

for some $C$ independent of $M$, as well as

$$
\begin{gathered}
\sum_{0 \leq|\beta| \leq 2}\left\|P_{k} \Gamma^{\beta} \psi_{\alpha, 1, j+1}\right\|_{S[k]\left([-T, T] \times \mathbb{R}^{3}\right)} \leq\left[\epsilon \tilde{M} 2^{C \delta l}+C 2^{C \delta l}\right] c_{k}, \forall k \in \mathbb{Z} \\
\left\|P_{k} \nabla_{x}^{N} \psi_{\alpha, 1, j+1}\right\|_{S[k]\left([-T, T] \times \mathbb{R}^{3}\right)} \leq\left[\epsilon M_{N}+M_{N-1}\right] d_{k}, \forall k \in \mathbb{Z}
\end{gathered}
$$

Furthermore, we obtain for the differences

$$
\sum_{0 \leq|\beta| \leq 2} \sum_{0 \leq k \leq N}\left\|\nabla_{x}^{k} \Gamma^{\beta} P_{k}\left[\psi_{\alpha, 1, j+1}-\psi_{\alpha, 1, j}\right]\right\|_{S[k]\left([-T, T] \times \mathbb{R}^{3}\right)} \leq C_{N} \epsilon^{j}
$$

To see how the proof of Proposition 2.1 follows from this, note that each $\nabla_{x}^{k} \Gamma^{\beta} P_{k}\left[\psi_{\alpha, 1, j}\right]$ converges with respect to $L_{t}^{\infty} \dot{H}^{\frac{1}{2}}$, whence the limit is smooth and satisfies the same bounds.

Hence we now direct our efforts to proving Proposition 4.5. This will be achieved via multilinear estimates much in the spirit of $[\mathbf{9}],[\mathbf{1 1}]$, the only new ingredient being the $\Gamma^{\beta}$ :

Proof. (Proposition 4.5) We shall first establish the inequality

$$
\left\|P_{k} \psi_{\alpha, 1, j+1}\right\|_{S[k]\left([-T, T] \times \mathbb{R}^{3}\right)} \leq[\epsilon M+C] d_{k}, \forall k \in \mathbb{Z}
$$

This will follow from the energy inequality Theorem 4.4 as well as the following two fundamental Propositions 4.6, 4.9. The first deals with estimating the trilinear null-forms on the right hand side of (2.6), while the 2 nd deals with the higher order terms

Proposition 4.6. The following trilinear null-form estimates hold:

$$
\begin{aligned}
& \left\|P_{k} \partial^{\beta}\left[R_{\alpha} P_{k_{1}} \psi P_{k_{4}} \triangle^{-1} \sum_{j=1}^{3} \partial_{j}\left(R_{\beta} P_{k_{2}} \psi_{2} R_{j} P_{k_{3}} \psi_{3}-R_{j} P_{k_{2}} \psi_{2} R_{\beta} P_{k_{3}} \psi_{3}\right)\right]\right\|_{N[k]} \\
& \lesssim 2^{-\delta_{1}\left|k_{2}-k_{3}\right|} 2^{\delta_{2}\left[k_{4}-\max \left\{k_{2}, k_{3}\right\}\right]} 2^{-\delta_{3}\left|k-k_{1}\right|} \prod_{i=1}^{3}\left\|P_{k_{i}} \psi_{i}\right\|_{S\left[k_{i}\right]} \\
& \left\|P_{k} \partial^{\beta}\left[R_{\beta} P_{k_{1}} \psi P_{k_{4}} \triangle^{-1} \sum_{j=1}^{3} \partial_{j}\left(R_{\alpha} P_{k_{2}} \psi_{2} R_{j} P_{k_{3}} \psi_{3}-R_{j} P_{k_{2}} \psi_{2} R_{\alpha} P_{k_{3}} \psi_{3}\right)\right]\right\|_{N[k]} \\
& \lesssim 2^{-\delta_{1}\left|k_{2}-k_{3}\right|} 2^{\delta_{2}\left[k_{4}-\max \left\{k_{2}, k_{3}\right\}\right]} 2^{-\delta_{3}\left|k-k_{1}\right|} \prod_{i=1}^{3}\left\|P_{k_{i}} \psi_{i}\right\|_{S\left[k_{i}\right]}
\end{aligned}
$$




$$
\begin{aligned}
& \left\|P_{k} \partial^{\alpha}\left[R_{\nu} P_{k_{1}} \psi P_{k_{4}} \triangle^{-1} \sum_{j=1}^{3} \partial_{j}\left(R^{\nu} P_{k_{2}} \psi_{2} R_{j} P_{k_{3}} \psi_{3}-R_{j} P_{k_{2}} \psi_{2} R^{\nu} P_{k_{3}} \psi_{3}\right)\right]\right\|_{N[k]} \\
& \lesssim 2^{-\delta_{1}\left|k_{2}-k_{3}\right|} 2^{\delta_{2}\left[k_{4}-\max \left\{k_{2}, k_{3}\right\}\right]} 2^{-\delta_{3}\left|k-k_{1}\right|} \prod_{i=1}^{3}\left\|P_{k_{i}} \psi_{i}\right\|_{S\left[k_{i}\right]}
\end{aligned}
$$

for suitable positive $\delta_{1,2,3}$.

REMARK 4.7. We observe that these estimates are very similar to Theorem 4.2 contained in [10], and indeed essentially implicitly contained in the proofs of [10], see also [11] in the 2-dmensional context. The only extra feature here is an exponential gain in the difference $k_{4}-\max \left\{k_{2}, k_{3}\right\}$. Our treatment shall be correspondingly brief.

Proof. (Proposition 4.6) We shall treat the first inequality, the other two following from identical reasoning. By scaling invariance we may assume $k=0$. We note that the cases $k_{4}=k_{2}+O(1), k_{1} \in[-10,10]^{c}$ follow from Theorem 4.2 in [10]. Hence we now assume $k_{2}=k_{3}+O(1)>>k_{4}, k_{1} \in[-10,10], k_{4}<15$. One distinguishes between the following cases:

(1): output in elliptic regime. This is the expression

$$
P_{0} Q_{>20} \partial^{\beta}\left[R_{\alpha} P_{k_{1}} \psi P_{k_{4}} \triangle^{-1} \sum_{j=1}^{3} \partial_{j}\left(R_{\beta} P_{k_{2}} \psi_{2} R_{j} P_{k_{3}} \psi_{3}-R_{j} P_{k_{2}} \psi_{2} R_{\beta} P_{k_{3}} \psi_{3}\right)\right]
$$

Recalling the definition of $\|\cdot\|_{N[0]}$, we need to estimate this with respect to

$$
\|\cdot\|_{\dot{X}_{0}^{-\frac{1}{2},-\frac{1}{2}, \infty} \cap \square\left|\nabla_{x, t}\right|^{-1} L_{t}^{\infty} \dot{H}_{x}^{-\frac{1}{2}}}
$$

First, we observe easily that

$$
\begin{aligned}
& \| \square^{-1}\left|\nabla_{x, t}\right| P_{0} Q_{>20} \partial^{\beta}\left[R_{\alpha} P_{k_{1}} \psi\right. \\
& \left.P_{k_{4}} \triangle^{-1} \sum_{j=1}^{3} \partial_{j}\left(R_{\beta} P_{k_{2}} \psi_{2} R_{j} P_{k_{3}} \psi_{3}-R_{j} P_{k_{2}} \psi_{2} R_{\beta} P_{k_{3}} \psi_{3}\right)\right] \|_{L_{t}^{\infty} \dot{H}^{-\frac{1}{2}}} \\
& \lesssim\left\|R_{\alpha} P_{k_{1}} \psi\right\|_{L_{t}^{\infty} L_{x}^{2}}\left\|P_{k_{4}} \triangle^{-1} \sum_{j=1}^{3} \partial_{j}\left(R_{\beta} P_{k_{2}} \psi_{2} R_{j} P_{k_{3}} \psi_{3}-R_{j} P_{k_{2}} \psi_{2} R_{\beta} P_{k_{3}} \psi_{3}\right)\right\|_{L_{t}^{\infty} L_{x}^{\infty}}
\end{aligned}
$$

which can be bounded by $\lesssim 2^{k_{4}-k_{2}} \prod_{i=1}^{3}\left\|P_{k_{i}} \psi_{i}\right\|_{S\left[k_{i}\right]}$, which is as desired. Next, freeze the modulation of the output to dyadic size $2^{l}, l>20$. Then we can write (using that $k_{1} \in[-10,10]$ )

$$
\begin{aligned}
& P_{0} Q_{l} \partial^{\beta}\left[R_{\alpha} P_{k_{1}} \psi P_{k_{4}} \triangle^{-1} \sum_{j=1}^{3} \partial_{j}\left(R_{\beta} P_{k_{2}} \psi_{2} R_{j} P_{k_{3}} \psi_{3}-R_{j} P_{k_{2}} \psi_{2} R_{\beta} P_{k_{3}} \psi_{3}\right)\right] \\
& =P_{0} Q_{l} \partial^{\beta}\left[R_{\alpha} P_{k_{1}} Q_{\geq l-10} \psi P_{k_{4}} \triangle^{-1} \sum_{j=1}^{3} \partial_{j}\left(R_{\beta} P_{k_{2}} \psi_{2} R_{j} P_{k_{3}} \psi_{3}-R_{j} P_{k_{2}} \psi_{2} R_{\beta} P_{k_{3}} \psi_{3}\right)\right] \\
& +P_{0} Q_{l} \partial^{\beta}\left[R_{\alpha} P_{k_{1}} Q_{<l-10} \psi\right. \\
& \left.P_{k_{4}} Q_{>l-10} \triangle^{-1} \sum_{j=1}^{3} \partial_{j}\left(R_{\beta} P_{k_{2}} \psi_{2} R_{j} P_{k_{3}} \psi_{3}-R_{j} P_{k_{2}} \psi_{2} R_{\beta} P_{k_{3}} \psi_{3}\right)\right]
\end{aligned}
$$


The first term on the right is estimated by

$$
\begin{aligned}
& \| P_{0} Q_{l} \partial^{\beta}\left[R_{\alpha} P_{k_{1}} Q_{\geq l-10} \psi\right. \\
& \left.P_{k_{4}} \triangle^{-1} \sum_{j=1}^{3} \partial_{j}\left(R_{\beta} P_{k_{2}} \psi_{2} R_{j} P_{k_{3}} \psi_{3}-R_{j} P_{k_{2}} \psi_{2} R_{\beta} P_{k_{3}} \psi_{3}\right)\right] \|_{\dot{X}_{0}^{-\frac{1}{2},-\frac{1}{2}, \infty}} \\
& =\sum_{l_{1} \geq l-10} \| P_{0} Q_{l} \partial^{\beta}\left[R_{\alpha} P_{k_{1}} Q_{l_{1}} \psi\right. \\
& \left.P_{k_{4}} \triangle^{-1} \sum_{j=1}^{3} \partial_{j}\left(R_{\beta} P_{k_{2}} \psi_{2} R_{j} P_{k_{3}} \psi_{3}-R_{j} P_{k_{2}} \psi_{2} R_{\beta} P_{k_{3}} \psi_{3}\right)\right] \|_{\dot{X}_{0}^{-\frac{1}{2},-\frac{1}{2}, \infty}} \\
& \lesssim \sum_{l_{1}>10} 2^{\frac{1}{2}\left(l-l_{1}\right)} 2^{k_{4}-k_{2}} \prod_{i=1}^{3}\left\|P_{k_{i}} \psi_{i}\right\|_{S\left[k_{i}\right]} \lesssim 2^{k_{4}-k_{2}} \prod_{i=1}^{3}\left\|P_{k_{i}} \psi_{i}\right\|_{S\left[k_{i}\right]}
\end{aligned}
$$

For the 2nd term, we use the following

LEMMA 4.8. If $l>>k$, then the following bound holds for suitable $\delta>0$ :

$$
\left\|P_{k} Q_{l}\left[R_{\nu} P_{k_{1}} \psi_{1} P_{k_{2}} \psi_{2}\right]\right\|_{L_{t}^{2} L_{x}^{2}} \lesssim 2^{\frac{k-l}{2}} 2^{\delta\left[\min \left\{k, k_{1}, k_{2}\right\}-\max \left\{k, k_{1}, k_{2}\right\}\right]} \prod_{i=1,2}\left\|P_{k_{i}} \psi_{i}\right\|_{S\left[k_{i}\right]}
$$

Proof. It is very similar to that of the last part of lemma 4.2: we can write

$$
\begin{aligned}
& P_{k} Q_{l}\left[R_{\nu} P_{k_{1}} \psi_{1} P_{k_{2}} \psi_{2}\right]= \\
& P_{k} Q_{l}\left[R_{\nu} P_{k_{1}} Q_{\geq l-10} \psi_{1} P_{k_{2}} \psi_{2}\right] \\
& +P_{k} Q_{l}\left[R_{\nu} P_{k_{1}} Q_{<l-10} \psi_{1} P_{k_{2}} Q_{\geq l-10} \psi_{2}\right] \\
& +P_{k} Q_{l}\left[R_{\nu} P_{k_{1}} Q_{<l-10} \psi_{1} P_{k_{2}} Q_{<l-10} \psi_{2}\right]
\end{aligned}
$$

Here the last term on the right is nonzero only if $k_{1}=l+O(1)$. Then when $k_{1}=k_{2}+O(1)$ we estimate

$$
\begin{aligned}
& \left\|P_{k} Q_{l}\left[R_{\nu} P_{k_{1}} Q_{\geq l-10} \psi_{1} P_{k_{2}} \psi_{2}\right]\right\|_{L_{t}^{2} L_{x}^{2}} \lesssim 2^{\frac{3 k}{2}}\left\|R_{\nu} P_{k_{1}} Q_{\geq l-10} \psi_{1}\right\|_{L_{t}^{2} L_{x}^{2}}\left\|P_{k_{2}} \psi_{2}\right\|_{L_{t}^{\infty} L_{x}^{2}} \\
& \lesssim 2^{\frac{3 k}{2}-\frac{1}{2} l-k_{1}} \prod_{i=, 2}\left\|P_{k_{i}} \psi_{i}\right\|_{S\left[k_{i}\right]},
\end{aligned}
$$

which is as desired. The cases $k=k_{1}+O(1), k=k_{2}+O(1)$ are handled analogously, as is the expression $P_{k} Q_{l}\left[R_{\nu} P_{k_{1}} Q_{<l-10} \psi_{1} P_{k_{2}} Q_{\geq l-10} \psi_{2}\right]$. Now for the last term above, we may assume $k_{1} \in[l-5, l+5]$. Furthermore, we may microlocalize the two inputs to the same half-space $\pm \tau>0$, i. e.

$$
P_{k} Q_{l}\left[R_{\nu} P_{k_{1}} Q_{<l-10} \psi_{1} P_{k_{2}} Q_{<l-10} \psi_{2}\right]=\sum_{ \pm} P_{k} Q_{l}^{ \pm}\left[R_{\nu} P_{k_{1}} Q_{<l-10}^{ \pm} \psi_{1} P_{k_{2}} Q_{<l-10}^{ \pm} \psi_{2}\right]
$$

We split this into three terms as follows:

$$
\begin{aligned}
& \sum_{ \pm} P_{k} Q_{l}^{ \pm}\left[R_{\nu} P_{k_{1}} Q_{<l-10}^{ \pm} \psi_{1} P_{k_{2}} Q_{<l-10}^{ \pm} \psi_{2}\right] \\
& =\sum_{ \pm} P_{k} Q_{l}^{ \pm}\left[R_{\nu} P_{k_{1}} Q_{\left[\frac{3}{2} k-\frac{1}{2} k_{1}, l-10\right]}^{ \pm} \psi_{1} P_{k_{2}} Q_{<l-10}^{ \pm} \psi_{2}\right] \\
& +\sum_{ \pm} P_{k} Q_{l}^{ \pm}\left[R_{\nu} P_{k_{1}} Q_{<\frac{3}{2} k-\frac{1}{2} k_{1}}^{ \pm} \psi_{1} P_{k_{2}} Q_{\left[\frac{3}{2} k-\frac{1}{2} k_{1}, l-10\right]}^{ \pm} \psi_{2}\right] \\
& +\sum_{ \pm} P_{k} Q_{l}^{ \pm}\left[R_{\nu} P_{k_{1}} Q_{<\frac{3}{2} k-\frac{1}{2} k_{1}}^{ \pm} \psi_{1} P_{k_{2}} Q_{<\frac{3}{2} k-\frac{1}{2} k_{1}}^{ \pm} \psi_{2}\right]
\end{aligned}
$$


The first term is estimated by

$$
\begin{aligned}
& \left\|\sum_{ \pm} P_{k} Q_{l}^{ \pm}\left[R_{\nu} P_{k_{1}} Q_{\left[\frac{3}{2} k-\frac{1}{2} k_{1}, l-10\right]}^{ \pm} \psi_{1} P_{k_{2}} Q_{<l-10}^{ \pm} \psi_{2}\right]\right\|_{L_{t}^{2} L_{x}^{2}} \\
& \lesssim \sum_{ \pm} 2^{\frac{3}{2} k} 2^{-\frac{k_{1}}{2}-\frac{\frac{3}{2} k-\frac{1}{2} k_{1}}{2}}\left\|R_{\nu} P_{k_{1}} Q_{\left[\frac{3}{2} k-\frac{1}{2} k_{1}, l-10\right]}^{ \pm} \psi_{1}\right\|_{\dot{X}_{k_{1}}^{\frac{1}{2}, \frac{1}{2}, \infty}}\left\|P_{k_{2}} Q_{<l-10}^{ \pm} \psi_{2}\right\|_{L_{t}^{\infty} L_{x}^{2}} \\
& \lesssim 2^{\frac{3}{2} k-k_{1}-\frac{\frac{3}{2} k-\frac{1}{2} k_{1}}{2}} \prod_{i=1,2}\left\|P_{k_{i}} \psi_{i}\right\|_{S\left[k_{i}\right]}=2^{\frac{3}{4}\left(k-k_{1}\right)} \prod_{i=1,2}\left\|P_{k_{i}} \psi_{i}\right\|_{S\left[k_{i}\right]}
\end{aligned}
$$

The 2nd term above can be estimated similarly. Finally, for the third term, we can decompose it into

$$
\begin{aligned}
& \sum_{ \pm} P_{k} Q_{l}^{ \pm}\left[R_{\nu} P_{k_{1}} Q_{<\frac{3}{2} k-\frac{1}{2} k_{1}}^{ \pm} \psi_{1} P_{k_{2}} Q_{<\frac{3}{2} k-\frac{1}{2} k_{1}}^{ \pm} \psi_{2}\right] \\
& =\sum_{ \pm} \sum_{\substack{\kappa_{1,2} \in K_{\frac{3}{4}\left(k-k_{1}\right)} \mid \operatorname{dist}\left(\kappa_{1},-\kappa_{2}\right) \lesssim 2 \frac{3}{4}\left(k-k_{1}\right) \\
P_{k} Q_{l}^{ \pm}\left[R_{\nu} P_{k_{1}, \kappa_{1}} Q_{<\frac{3}{2} k-\frac{1}{2} k_{1}}^{ \pm} \psi_{1} P_{k_{2}, \kappa_{2}} Q_{<\frac{3}{2} k-\frac{1}{2} k_{1}}^{ \pm} \psi_{2}\right]}}
\end{aligned}
$$

Using the definition of $\|\cdot\|_{S[k, \kappa]}$ and the Cauchy-Schwarz inequality, we can estimate this by

$$
\begin{aligned}
& \| \sum_{ \pm} \sum_{\kappa_{1,2} \in K_{\frac{3}{4}\left(k-k_{1}\right)} \mid \operatorname{dist}\left(\kappa_{1},-\kappa_{2}\right) \lesssim 22^{\frac{3}{4}\left(k-k_{1}\right)}} \\
& P_{k} Q_{l}^{ \pm}\left[R_{\nu} P_{k_{1}, \kappa_{1}} Q_{<\frac{3}{2} k-\frac{1}{2} k_{1}}^{ \pm} \psi_{1} P_{k_{2}, \kappa_{2}} Q_{<\frac{3}{2} k-\frac{1}{2} k_{1}}^{ \pm} \psi_{2}\right] \mid \|_{L_{t}^{2} L_{x}^{2}} \\
& \left.\lesssim 2^{\frac{3}{4}\left(k-k_{1}\right)} \sum_{\kappa \in K_{\frac{3}{4}\left(k-k_{1}\right)}}\left\|P_{k_{1}, \kappa} Q_{<\frac{3}{2} k-\frac{1}{2} k_{1}}^{ \pm} \psi_{1}\right\|_{S\left[k_{1}, \kappa_{1}\right]}^{2}\right)^{\frac{1}{2}} \\
& \times\left(\sum_{\kappa \in K_{\frac{3}{4}\left(k-k_{1}\right)}}\left\|P_{k_{2}, \kappa} Q_{<\frac{3}{2} k-\frac{1}{2} k_{1}}^{ \pm} \psi_{1}\right\|_{S\left[k_{1}, \kappa_{1}\right]}^{2}\right)^{\frac{1}{2}}
\end{aligned}
$$

This in turn is bounded by $\lesssim 2^{\frac{3}{4}\left(k-k_{1}\right)} \prod_{i=1,2}\left\|P_{k_{i}} \psi_{i}\right\|_{S\left[k_{i}\right]}$, which is as desired.

Then we can estimate

$$
\begin{aligned}
& \| P_{0} Q_{l} \partial^{\beta}\left[R_{\alpha} P_{k_{1}} Q_{<l-10} \psi\right. \\
& \left.\quad P_{k_{4}} Q_{>l-10} \triangle^{-1} \sum_{j=1}^{3} \partial_{j}\left(R_{\beta} P_{k_{2}} \psi_{2} R_{j} P_{k_{3}} \psi_{3}-R_{j} P_{k_{2}} \psi_{2} R_{\beta} P_{k_{3}} \psi_{3}\right)\right] \|_{\dot{X}_{0}^{-\frac{1}{2},-\frac{1}{2}, \infty}} \\
& \lesssim 2^{\frac{l}{2}}\left\|R_{\alpha} P_{k_{1}} Q_{<l-10} \psi\right\|_{L_{t}^{\infty} L_{x}^{2}} \\
& \left.\quad \| P_{k_{4}} Q_{>l-10} \triangle^{-1} \sum_{j=1}^{3} \partial_{j}\left(R_{\beta} P_{k_{2}} \psi_{2} R_{j} P_{k_{3}} \psi_{3}-R_{j} P_{k_{2}} \psi_{2} R_{\beta} P_{k_{3}} \psi_{3}\right)\right] \|_{L_{t}^{2} L_{x}^{\infty}} \\
& \lesssim 2^{\frac{k_{4}}{2}} 2^{\delta\left[k_{4}-k_{2}\right]} \prod_{i=1}^{3}\left\|P_{k_{i}} \psi_{i}\right\|_{S\left[k_{i}\right]}
\end{aligned}
$$

This concludes estimating the contribution of the output in the elliptic regime. 
(2): Output in hyperbolic regime. This is the expression

$$
P_{0} Q_{<20} \partial^{\beta}\left[R_{\alpha} P_{k_{1}} \psi P_{k_{4}} \triangle^{-1} \sum_{j=1}^{3} \partial_{j}\left(R_{\beta} P_{k_{2}} \psi_{2} R_{j} P_{k_{3}} \psi_{3}-R_{j} P_{k_{2}} \psi_{2} R_{\beta} P_{k_{3}} \psi_{3}\right)\right]
$$

Here, we first reduce $R_{\alpha} P_{k_{1}} \psi$ as well as the output further to modulation $<2^{k_{4}-10}$. To achieve this, estimate

$$
\begin{aligned}
& \| P_{0} Q_{20>. \geq k_{4}-10} \partial^{\beta}\left[R_{\alpha} P_{k_{1}} \psi_{1}\right. \\
& \left.P_{k_{4}} \triangle^{-1} \sum_{j=1}^{3} \partial_{j}\left(R_{\beta} P_{k_{2}} \psi_{2} R_{j} P_{k_{3}} \psi_{3}-R_{j} P_{k_{2}} \psi_{2} R_{\beta} P_{k_{3}} \psi_{3}\right)\right] \|_{\dot{X}_{0}^{-\frac{1}{2},-\frac{1}{2}, 1}} \\
& \lesssim 2^{-\frac{k_{4}}{2}}\left\|R_{\alpha} P_{k_{1}} \psi_{1}\right\|_{L_{t}^{\infty} L_{x}^{2}} \\
& \left\|P_{k_{4}} \triangle^{-1} \sum_{j=1}^{3} \partial_{j}\left(R_{\beta} P_{k_{2}} \psi_{2} R_{j} P_{k_{3}} \psi_{3}-R_{j} P_{k_{2}} \psi_{2} R_{\beta} P_{k_{3}} \psi_{3}\right)\right\|_{L_{t}^{2} L_{x}^{\infty}}
\end{aligned}
$$

Using Lemma 4.2, the right hand factor can be estimated by

$$
\begin{aligned}
& \left\|P_{k_{4}} \triangle^{-1} \sum_{j=1}^{3} \partial_{j}\left(R_{\beta} P_{k_{2}} \psi_{2} R_{j} P_{k_{3}} \psi_{3}-R_{j} P_{k_{2}} \psi_{2} R_{\beta} P_{k_{3}} \psi_{3}\right)\right\|_{L_{t}^{2} L_{x}^{\infty}} \\
& \lesssim 2^{\frac{k_{4}}{2}} 2^{\frac{k_{4}-k_{2}}{2}} \prod_{i=2,3}\left\|P_{k_{i}} \psi_{i}\right\|_{S\left[k_{i}\right]}
\end{aligned}
$$

and the desired estimate follows. The expression

$P_{0} Q_{<20} \partial^{\beta}\left[R_{\alpha} P_{k_{1}} Q_{\geq k_{4}-10} \psi_{1} P_{k_{4}} \triangle^{-1} \sum_{j=1}^{3} \partial_{j}\left(R_{\beta} P_{k_{2}} \psi_{2} R_{j} P_{k_{3}} \psi_{3}-R_{j} P_{k_{2}} \psi_{2} R_{\beta} P_{k_{3}} \psi_{3}\right)\right]$

is estimated similarly(place the output into $L_{t}^{1} \dot{H}^{-\frac{1}{2}}$ ), hence we now need to estimate

$P_{0} Q_{<k_{4}-10} \partial^{\beta}\left[R_{\alpha} P_{k_{1}} Q_{<k_{4}-10} \psi_{1} P_{k_{4}} \triangle^{-1} \sum_{j=1}^{3} \partial_{j}\left(R_{\beta} P_{k_{2}} \psi_{2} R_{j} P_{k_{3}} \psi_{3}-R_{j} P_{k_{2}} \psi_{2} R_{\beta} P_{k_{3}} \psi_{3}\right)\right]$.

Note that we may include an operator $Q_{<k_{4}+10}$ in front of $P_{k_{4}} \triangle^{-1} \ldots$. We further reduce the inner inputs $P_{k_{2,3}} \psi_{2,3}$ to modulation $<2^{k_{4}+20}$ as follows: for example, consider

$$
\begin{aligned}
& P_{0} Q_{<k_{4}-10} \partial^{\beta}\left[R_{\alpha} P_{k_{1}} Q_{<k_{4}-10} \psi_{1}\right. \\
& \left.P_{k_{4}} \triangle^{-1} \sum_{j=1}^{3} \partial_{j}\left(R_{\beta} P_{k_{2}} Q_{\geq k_{4}+20} \psi_{2} R_{j} P_{k_{3}} \psi_{3}-R_{j} P_{k_{2}} Q_{\geq k_{4}+20} \psi_{2} R_{\beta} P_{k_{3}} \psi_{3}\right)\right]
\end{aligned}
$$


Simple geometric reasoning then allows one to include a multiplier $Q_{>k_{4}+15}$ in front of each $P_{k_{3}} \psi_{3}$. Then one estimates

$$
\begin{aligned}
& \| P_{0} Q_{<k_{4}-10} \partial^{\beta}\left[R _ { \alpha } P _ { k _ { 1 } } Q _ { < k _ { 4 } - 1 0 } \psi _ { 1 } P _ { k _ { 4 } } \triangle ^ { - 1 } \sum _ { j = 1 } ^ { 3 } \partial _ { j } \left(R_{\beta} P_{k_{2}} Q_{\geq k_{4}+20} \psi_{2} R_{j} P_{k_{3}} Q_{>k_{4}+15} \psi_{3}\right.\right. \\
& \left.\left.-R_{j} P_{k_{2}} Q_{\geq k_{4}+20} \psi_{2} R_{\beta} P_{k_{3}} Q_{>k_{4}+15} \psi_{3}\right)\right]\left.\right|_{L_{t}^{1} \dot{H}_{x}^{-\frac{1}{2}}} \\
& \lesssim 2^{2 k_{4}}|| R_{\alpha} P_{k_{1}} Q_{<k_{4}-10} \psi_{1} \|_{L_{t}^{\infty} L_{x}^{2}} \\
& \left\|\nabla_{x, t} \nabla^{-1} P_{k_{2}} Q_{\geq k_{4}+20} \psi_{2}\right\|_{L_{t}^{2} L_{x}^{2}}\left\|\nabla_{x, t} \nabla^{-1} P_{k_{3}} Q_{\geq k_{4}+20} \psi_{3}\right\|_{L_{t}^{2} L_{x}^{2}} \\
& \lesssim 2^{k_{4}-k_{2}} \prod_{i=1}^{3}\left\|P_{k_{i}} \psi_{i}\right\|_{S\left[k_{i}\right]}
\end{aligned}
$$

We now let the outer derivative fall inside and obtain two terms, each of which admits a null-form expansion: the terms are

$$
\begin{aligned}
& P_{0} Q_{<k_{4}-10}\left[R _ { \alpha } \partial ^ { \beta } P _ { k _ { 1 } } Q _ { < k _ { 4 } - 1 0 } \psi _ { 1 } P _ { k _ { 4 } } \triangle ^ { - 1 } \sum _ { j = 1 } ^ { 3 } \partial _ { j } \left(R_{\beta} P_{k_{2}} Q_{<k_{4}+20} \psi_{2} R_{j} P_{k_{3}} Q_{<k_{4}+20} \psi_{3}\right.\right. \\
& \left.\left.-R_{j} P_{k_{2}} Q_{<k_{4}+20} \psi_{2} R_{\beta} P_{k_{3}} Q_{<k_{4}+20} \psi_{3}\right)\right]
\end{aligned}
$$

$$
\begin{aligned}
& P_{0} Q_{<k_{4}-10}\left[R _ { \alpha } P _ { k _ { 1 } } Q _ { < k _ { 4 } - 1 0 } \psi _ { 1 } P _ { k _ { 4 } } \triangle ^ { - 1 } \sum _ { j = 1 } ^ { 3 } \partial _ { j } \partial ^ { \beta } \left(R_{\beta} P_{k_{2}} Q_{<k_{4}+20} \psi_{2} R_{j} P_{k_{3}} Q_{<k_{4}+20} \psi_{3}\right.\right. \\
& \left.\left.-R_{j} P_{k_{2}} Q_{<k_{4}+20} \psi_{2} R_{\beta} P_{k_{3}} Q_{<k_{4}+20} \psi_{3}\right)\right]
\end{aligned}
$$

We treat these separately. In order to streamline the formulae a bit, we shall omit the localizers $Q_{<k_{4}+20}$, it being understood that the inputs $P_{k_{2,3}} \psi_{2,3}$ have Fourier support at distance $<2^{k_{4}+20}$ from the light cone.

(3): The first null-form. We use the expansion

$$
\begin{aligned}
& 2 \sum_{j=1}^{3} \triangle^{-1} \partial_{j}\left[R_{\nu} f R_{j} g-R_{j} f R_{\nu} f\right] \partial^{\nu} h \\
& =\sum_{j=1}^{3} \square\left[\triangle^{-1} \partial_{j}\left[\nabla^{-1} f R_{j} g\right] h\right]-\sum_{j=1}^{3} \square \triangle^{-1} \partial_{j}\left[\nabla^{-1} f R_{j} g\right] h \\
& -\sum_{j=1}^{3} \triangle^{-1} \partial_{j}\left[\nabla^{-1} f R_{j} g\right] \square h-\nabla^{-1} f \square\left[\left(\nabla^{-1} g\right) h\right] \\
& +\nabla^{-1} f \square\left(\nabla^{-1} g\right) h+\nabla^{-1} f \nabla^{-1} g \square h
\end{aligned}
$$

Hence we need to estimate the following terms: first assume $k_{2}<-20$. 
(i): $P_{0} Q_{<k_{4}-10} \square\left[R_{\alpha} P_{k_{1}} Q_{<k_{4}-10} \psi_{1} P_{k_{4}} \triangle^{-1} \sum_{j=1}^{3} \partial_{j}\left(\nabla^{-1} P_{k_{2}} \psi_{2} R_{j} P_{k_{3}} \psi_{3}\right)\right]$. Here we use lemma 4.1 to estimate it by

$$
\begin{aligned}
& \left\|P_{0} Q_{<k_{4}-10} \square\left[R_{\alpha} P_{k_{1}} Q_{<k_{4}-10} \psi_{1} P_{k_{4}} \triangle^{-1} \sum_{j=1}^{3} \partial_{j}\left(\nabla^{-1} P_{k_{2}} \psi_{2} R_{j} P_{k_{3}} \psi_{3}\right)\right]\right\|_{\dot{X}_{0}^{-\frac{1}{2},-\frac{1}{2}, 1}} \\
& \lesssim 2^{\frac{k_{4}}{2}}\left\|R_{\alpha} P_{k_{1}} Q_{<k_{4}-10} \psi_{1}\right\|_{L_{t}^{\infty} L_{x}^{2}}\|\| P_{k_{4}} \triangle^{-1} \sum_{j=1}^{3} \partial_{j}\left(\nabla^{-1} P_{k_{2}} \psi_{2} R_{j} P_{k_{3}} \psi_{3}\right) \|_{L_{t}^{2} L_{x}^{\infty}} \\
& \lesssim 2^{-\frac{k_{4}}{2}-k_{2}} 2^{\frac{6}{p} k_{4}}|| R_{\alpha} P_{k_{1}} Q_{<k_{4}-10} \psi_{1}\left\|_{L_{t}^{\infty} L_{x}^{2}}\right\| P_{k_{2}} \psi_{2}\left\|_{L_{t}^{4} L_{x}^{p}}\right\| P_{k_{3}} \psi_{3} \|_{L_{t}^{4} L_{x}^{p}}
\end{aligned}
$$

This is seen to be bounded by $\lesssim 2^{\delta\left[k_{4}-k_{2}\right]} \prod_{i=1}^{3}\left\|P_{k_{i}} \psi_{i}\right\|_{S\left[k_{i}\right]}$ for some $\delta>0$, provided we choose $p>4$ sufficiently close to 4 .

(ii): $P_{0} Q_{<k_{4}-10}\left[R_{\alpha} P_{k_{1}} Q_{<k_{4}-10} \psi_{1} P_{k_{4}} \triangle^{-1} \sum_{j=1}^{3} \partial_{j} \square\left(\nabla^{-1} P_{k_{2}} \psi_{2} R_{j} P_{k_{3}} \psi_{3}\right)\right]$. This we estimate by using Lemma 4.3 as well as Lemma 4.2: First, we have

$$
\begin{aligned}
& P_{0} Q_{<k_{4}-10}\left[R_{\alpha} P_{k_{1}} Q_{<k_{4}-10} \psi_{1} P_{k_{4}} \triangle^{-1} \sum_{j=1}^{3} \partial_{j} \square\left(\nabla^{-1} P_{k_{2}} \psi_{2} R_{j} P_{k_{3}} \psi_{3}\right)\right] \\
& =P_{0} Q_{<k_{4}-10}\left[R_{\alpha} P_{k_{1}} Q_{<k_{4}-10} \psi_{1} P_{k_{4}} Q_{<k_{4}+10} \triangle^{-1} \sum_{j=1}^{3} \partial_{j} \square\left(\nabla^{-1} P_{k_{2}} \psi_{2} R_{j} P_{k_{3}} \psi_{3}\right)\right]
\end{aligned}
$$

Then we first remove the localization operators $Q_{<k_{4}-10}$, which is simple (estimating as before) and omitted. Thus we now need to estimate (including an operator $Q_{<k_{1}}$ to render the $R_{\alpha}$ harmless)

$$
\begin{aligned}
& P_{0}\left[R_{\alpha} P_{k_{1}} Q_{<k_{1}} \psi_{1} P_{k_{4}} Q_{<k_{4}+10} \triangle^{-1} \sum_{j=1}^{3} \partial_{j} \square\left(\nabla^{-1} P_{k_{2}} \psi_{2} R_{j} P_{k_{3}} \psi_{3}\right)\right] \\
& =\sum_{j<k_{4}+10} P_{0}\left[R_{\alpha} P_{k_{1}} Q_{<k_{1}} \psi_{1} P_{k_{4}} Q_{j} \triangle^{-1} \sum_{j=1}^{3} \partial_{j} \square\left(\nabla^{-1} P_{k_{2}} \psi_{2} R_{j} P_{k_{3}} \psi_{3}\right)\right]
\end{aligned}
$$

Using Lemma 4.3 we have

$$
\begin{aligned}
& \left\|P_{0}\left[R_{\alpha} P_{k_{1}} Q_{<k_{1}} \psi_{1} P_{k_{4}} Q_{j} \triangle^{-1} \sum_{j=1}^{3} \partial_{j} \square\left(\nabla^{-1} P_{k_{2}} \psi_{2} R_{j} P_{k_{3}} \psi_{3}\right)\right]\right\|_{N[0]} \\
& \lesssim 2^{\delta\left(j-k_{4}\right)}\left\|P_{k_{1}} \psi_{1}\right\|_{S\left[k_{1}\right]}\left\|\square \nabla^{-1} P_{k_{4}} Q_{j}\left(\nabla^{-1} P_{k_{2}} \psi_{2} R_{j} P_{k_{3}} \psi_{3}\right)\right\|_{\dot{X}_{k_{4}}^{\frac{1}{2},-\frac{1}{2}, 1}}
\end{aligned}
$$

for suitable $\delta>0$. Using Lemma 4.2, we estimate this by

$$
\begin{aligned}
& 2^{\delta\left(j-k_{4}\right)}\left\|P_{k_{1}} \psi_{1}\right\|_{S\left[k_{1}\right]}\left\|\square \nabla^{-1} P_{k_{4}} Q_{j}\left(\nabla^{-1} P_{k_{2}} \psi_{2} R_{j} P_{k_{3}} \psi_{3}\right)\right\|_{\dot{X}_{k_{4}}^{\frac{1}{2},-\frac{1}{2}, 1}} \\
& \lesssim 2^{\delta\left(j-k_{4}\right)}\left\|P_{k_{1}} \psi_{1}\right\|_{S\left[k_{1}\right]} 2^{k_{4}-k_{2}} \prod_{i=1,2}\left\|P_{k_{i}} \psi_{i}\right\|_{S\left[k_{i}\right]}
\end{aligned}
$$

which is as desired upon summing over $j$.

(iii): $P_{0} Q_{<k_{4}-10}\left[R_{\alpha} \square P_{k_{1}} Q_{<k_{4}-10} \psi_{1} P_{k_{4}} \triangle^{-1} \sum_{j=1}^{3} \partial_{j}\left(\nabla^{-1} P_{k_{2}} \psi_{2} R_{j} P_{k_{3}} \psi_{3}\right)\right]$. This is estimated similarly to (i). Simply place the output into $L_{t}^{1} \dot{H}^{-\frac{1}{2}}$. 
(iv): the term corresponding to $\nabla^{-1} f \square\left(\nabla^{-1} g h\right)$ in the above expansion is the most difficult to handle; we use that $P_{k_{4}}$ acts via convolution with a function $a_{k_{4}}(x)$ of bounded $L^{1}$-mass. Further, we recall here the precise microlocalization of $P_{k_{2,3}} \psi_{2,3}$. Thus we write this term as

$$
\begin{gathered}
\int_{\mathbb{R}^{3}} a_{k_{4}}(y) P_{0} Q_{<k_{4}-10}\left[\square\left[P_{k_{1}} Q_{<k_{4}-10} R_{\alpha} \psi_{1} \nabla^{-1} P_{k_{3}} Q_{<k_{4}+20} \psi_{3}(.-y)\right]\right. \\
\left.\nabla^{-1} P_{k_{2}} Q_{<k_{4}+20} \psi_{2}(.-y)\right] d y
\end{gathered}
$$

Note that the restriction that $P_{k_{2}} \psi_{2} P_{k_{3}} \psi_{3}$ is reduced to frequency $2^{k_{4}}$ allows us to simultaneously localize them to angular sectors $\kappa_{2,3} \operatorname{such}$ that $\operatorname{dist}\left(\kappa_{2},-\kappa_{3}\right) \lesssim$ $2^{\frac{k_{4}-k_{2}}{2}}$. Thus we can write

$$
\begin{gathered}
\int_{\mathbb{R}^{3}} a_{k_{4}}(y) P_{0} Q_{<k_{4}-10}\left[\square\left[P_{k_{1}} Q_{<k_{4}-10} R_{\alpha} \psi_{1} \nabla^{-1} P_{k_{3}} Q_{<k_{4}+20} \psi_{3}(.-y)\right]\right. \\
\left.\nabla^{-1} P_{k_{2}} Q_{<k_{4}+20} \psi_{2}(.-y)\right] d y \\
\sum_{\kappa_{2,3} \in K_{k_{4}-k_{2}} \mid \operatorname{dist}\left(\kappa_{2},-\kappa_{3}\right) \lesssim 2^{k_{4}-k_{2}}}{ }_{\mathbb{R}^{3}} a_{k_{4}}(y) P_{0} Q_{<k_{4}-10}\left[\square\left[P_{k_{1}} Q_{<k_{4}-10} R_{\alpha} \psi_{1} \nabla^{-1} P_{k_{3}, \kappa_{3}} Q_{<k_{4}+20} \psi_{3}(.-y)\right]\right. \\
\left.\nabla^{-1} P_{k_{2}, \kappa_{2}} Q_{<k_{4}+20} \psi_{2}(.-y)\right] d y
\end{gathered}
$$

Here we first abolish the outer localizer $Q_{<k_{4}-10}$, and modify $\square$ to $\square Q_{<k_{3}+O(1)}$ : clearly, we have

$$
\begin{gathered}
P_{0} Q_{<k_{4}-10}\left[\square\left[P_{k_{1}} Q_{<k_{4}-10} R_{\alpha} \psi_{1} \nabla^{-1} P_{k_{3}, \kappa_{3}} Q_{<k_{4}+20} \psi_{3}(.-y)\right]\right. \\
\left.\nabla^{-1} P_{k_{2}, \kappa_{2}} Q_{<k_{4}+20} \psi_{2}(.-y)\right] \\
=P_{0} Q_{<k_{4}-10}\left[P_{k_{1}+O(1)} Q_{<k_{3}+O(1)} \square\left[P_{k_{1}} Q_{<k_{4}-10} R_{\alpha} \psi_{1} \nabla^{-1} P_{k_{3}, \kappa_{3}} Q_{<k_{4}+20} \psi_{3}(.-y)\right]\right. \\
\left.\nabla^{-1} P_{k_{2}, \kappa_{2}} Q_{<k_{4}+20} \psi_{2}(.-y)\right]
\end{gathered}
$$

Then, replacing the outer $Q_{<k_{4}-10}$ by $Q_{\geq k_{4}-10}$, say, we can estimate

$$
\begin{gathered}
\| P_{0} Q_{\geq k_{4}-10}\left[P _ { k _ { 1 } + O ( 1 ) } Q _ { < k _ { 3 } + O ( 1 ) } \square \left[P_{k_{1}} Q_{<k_{4}-10} R_{\alpha} \psi_{1}\right.\right. \\
\left.\left.\nabla^{-1} P_{k_{3}, \kappa_{3}} Q_{<k_{4}+20} \psi_{3}(.-y)\right] \nabla^{-1} P_{k_{2}, \kappa_{2}} Q_{<k_{4}+20} \psi_{2}(.-y)\right] \|_{\dot{X}_{0}^{-\frac{1}{2},-\frac{1}{2}, 1}} \\
\left.\left.\lesssim 2^{-\frac{k_{4}}{2}} 2^{k_{3}}\left\|P_{k_{1}} Q_{<k_{4}-10} R_{\alpha} \psi_{1}\right\|\right|_{L_{t}^{\infty} L_{x}^{2}}\left\|\nabla^{-1} P_{k_{3}, \kappa_{3}} Q_{<k_{4}+20} \psi_{3}(.-y)\right\|\right|_{L_{t}^{4} L_{x}^{\infty}} \\
\left\|\nabla^{-1} P_{k_{2}, \kappa_{2}} Q_{<k_{4}+20} \psi_{2}(.-y)\right\|_{L_{t}^{4} L_{x}^{\infty}}
\end{gathered}
$$

We have taken advantage of the fact (see e. g. [24]) that the operator $P_{k_{1}} Q_{<k_{3}+O(1)}$ acts boundedly on spaces of the form $L_{t}^{p} L_{x}^{2}, 1 \leq p \leq \infty$. Then we use Lemma 4.2 as well as the fact that

$$
\sum_{\kappa \in K_{l}}\left\|P_{k, \kappa} Q_{<k+2 l} \psi\right\|_{S[k]}^{2} \lesssim\left\|P_{k} \psi\right\|_{S[k]}^{2}
$$

This is straightforward for the first two components defining $\|\cdot\|_{S[k]}$. For the complicated null-frame part, use that $\|\cdot\|_{S\left[k, \kappa^{\prime}\right]} \lesssim\|\cdot\|_{S[k, \kappa]}$ for $\kappa \subset \kappa^{\prime}$. Hence we have 


$$
\begin{aligned}
\text { for }- & 10 \geq l^{\prime} \geq l \\
& \sum_{\kappa \in K_{l}} \sum_{\kappa^{\prime} \in K_{l^{\prime}}}\left\|P_{k, \kappa^{\prime}} Q_{<k+2 l^{\prime}}^{ \pm} P_{k, \kappa} Q_{<k+2 l} \psi\right\|_{S\left[k, \pm \kappa^{\prime}\right]}^{2} \\
& \lesssim \sum_{\kappa^{\prime} \in K_{l^{\prime}}} \sum_{\kappa \in K_{l}, \kappa \subset 2 \kappa^{\prime}}\left\|P_{k, \kappa^{\prime}} Q_{<k+2 l^{\prime}}^{ \pm} P_{k, \kappa} Q_{<k+2 l}^{ \pm} \psi\right\|_{S[k, \pm \kappa]}^{2} \\
& \lesssim \sum_{\kappa \in K_{l}} \sum_{\kappa^{\prime} \in K_{l^{\prime}} \mid \kappa \in 2 \kappa^{\prime}}\left\|P_{k, \kappa} Q_{<k+2 l}^{ \pm} \psi\right\|_{S[k, \pm \kappa]}^{2} \lesssim \sum_{\kappa \in K_{l}}\left\|P_{k, \kappa} Q_{<k+2 l}^{ \pm} \psi\right\|_{S[k, \pm \kappa]}^{2}
\end{aligned}
$$

The case when $l^{\prime}<l$ is handled similarly. Hence we now see that (using Bernstein's inequality)

$$
\begin{aligned}
& \sum_{\kappa_{1,2} \in K_{k_{4}-k_{2}} \mid \mathrm{dist}\left(\kappa_{2},-\kappa_{3}\right) \lesssim 2^{k_{4}-k_{2}}} \\
& 2^{-\frac{k_{4}}{2}} 2^{k_{3}}\left\|P_{k_{1}} Q_{<k_{4}-10} R_{\alpha} \psi_{1}\right\|_{L_{t}^{\infty} L_{x}^{2}}\left\|\nabla^{-1} P_{k_{3}, \kappa_{3}} Q_{<k_{4}+20} \psi_{3}(.-y)\right\|_{L_{t}^{4} L_{x}^{\infty}} \\
& \lesssim 2^{(1-)\left(k_{4}-k_{3}\right)} \prod_{i=1}^{3}\left\|\nabla^{-1} P_{k_{2}, \kappa_{2}} Q_{<k_{4}+20} \psi_{2}(.-y)\right\|_{L_{t}^{4} L_{x}^{\infty}}
\end{aligned}
$$

\section{We have now reduced ourselves to estimating}

$$
\begin{gathered}
\int_{\mathbb{R}^{3}} a_{k_{4}}(y) P_{0}\left[\square Q_{<k_{3}+O(1)}\left[P_{k_{1}} Q_{<k_{4}-10} R_{\alpha} \psi_{1} P_{k_{3}} Q_{<k_{4}+20} \psi_{3}(.-y)\right]\right. \\
\left.\nabla^{-1} P_{k_{2}} Q_{<k_{4}+20} \psi_{2}(.-y)\right] d y
\end{gathered}
$$

We first write

$$
\begin{aligned}
& \left\|\square P_{k_{1}+O(1)} Q_{<k_{3}+O(1)}\left[P_{k_{1}} Q_{<k_{4}-10} R_{\alpha} \psi_{1} \nabla^{-1} P_{k_{3}, \kappa_{3}} Q_{<k_{4}+20} \psi_{3}(.-y)\right]\right\|_{\dot{X}_{k_{1}}^{\frac{1}{2}, \frac{1}{2}, 1}} \\
& \lesssim \sum_{l<k_{3}+O(1)}\left\|P_{k_{1}+O(1)} Q_{l}\left[P_{k_{1}} Q_{<k_{4}-10} R_{\alpha} \psi_{1} \nabla^{-1} P_{k_{3}, \kappa_{3}} Q_{<k_{4}+20} \psi_{3}(.-y)\right]\right\|_{\dot{X}_{k_{1}}^{\frac{1}{2}, \frac{1}{2}, 1}}
\end{aligned}
$$

We need to estimate this expression for fixed $l$ first, the point being to eke out a small gain in $\left|\kappa_{3}\right|$. One distinguishes between different ranges for $l$ : first, assume $l \geq k_{4}+30$ whence $\frac{l-k_{3}}{2} \geq \frac{k_{4}-k_{3}}{2}+15$. Then write (here we have to go into full detail)

$$
\begin{aligned}
& P_{k_{1}+O(1)} Q_{l}\left[P_{k_{1}} Q_{<k_{4}-10} R_{\alpha} \psi_{1} \nabla^{-1} P_{k_{3}, \kappa_{3}} Q_{<k_{4}+20} \psi_{3}(.-y)\right] \\
& =\sum_{ \pm, \pm} \sum_{\kappa_{1,2}^{\prime} \in K_{\frac{l-k_{3}}{2}} \mid \operatorname{dist}\left( \pm \kappa_{1}^{\prime}, \pm \kappa_{2}^{\prime}\right) \sim 2^{\frac{l-k_{3}}{2}}} \\
& P_{k_{1}+O(1)} Q_{l}\left[P_{k_{1}, \kappa_{1}^{\prime}} Q_{<k_{4}-10}^{ \pm} R_{\alpha} \psi_{1} \nabla^{-1} P_{k_{3}, \kappa_{2}^{\prime}} P_{k_{3}, \kappa_{3}} Q_{<k_{4}+20}^{ \pm} \psi_{3}(.-y)\right],
\end{aligned}
$$

At this point, the operator $Q_{<k_{4}-10}^{ \pm}$becomes harmful, as it is applied to a large frequency input. However, we can easily abolish it, by estimating the contribution from $P_{k_{1}, \kappa_{1}^{\prime}} Q_{\geq k_{4}-10}^{ \pm} R_{\alpha} \psi_{1}$ as above, where the operator $Q_{\geq k_{4}-10}$ was applied to the output (here one places the output into $L_{t}^{1} \dot{H}^{-\frac{1}{2}}$ ). Now we estimate (Here $Q_{ \pm}$is 
the operator which localizes to $\pm \tau>0$ )

$$
\begin{aligned}
& \| \sum_{ \pm, \pm} \sum_{\kappa_{1,2}^{\prime} \in K_{\frac{l-k_{3}}{2}}} \sum_{\operatorname{dist}\left( \pm \kappa_{1}^{\prime}, \pm \kappa_{2}^{\prime}\right) \sim 2^{\frac{l-k_{3}}{2}}} \\
& P_{k_{1}+O(1)} Q_{l}\left[P_{k_{1}, \kappa_{1}^{\prime}} Q^{ \pm} R_{\alpha} \psi_{1} \nabla^{-1} P_{k_{3}, \kappa_{2}^{\prime}} P_{k_{3}, \kappa_{3}} Q_{<k_{4}+20}^{ \pm} \psi_{3}(.-y)\right] \|_{\dot{X}_{k_{1}}^{\frac{1}{2}, \frac{1}{2}, \infty}} \\
& \lesssim \sum_{\kappa_{1,2}^{\prime} \in K_{\frac{l-k_{3}}{2}} \mid \operatorname{dist}\left( \pm \kappa_{1}^{\prime}, \pm \kappa_{2}^{\prime}\right) \sim 2 \frac{l-k_{3}}{2}} \\
& 2^{\frac{l}{2}}\left\|P_{k_{1}, \kappa_{1}^{\prime}} Q^{ \pm} R_{\alpha} \psi_{1}\right\|_{N F A\left[ \pm \kappa_{2}^{\prime}\right]}\left\|\nabla^{-1} P_{k_{3}, \kappa_{2}^{\prime}} P_{k_{3}, \kappa_{3}} Q_{<k_{4}+20}^{ \pm} \psi_{3}\right\|_{P W\left[ \pm \kappa_{3}\right]}
\end{aligned}
$$

Since $\kappa_{2}^{\prime}$ is much larger than $\kappa_{3}$ in the present case, we only sum for finitely many $\kappa_{1,2}^{\prime}$ for fixed $\kappa_{3}$. Now we use the estimate

$$
\left\|P_{k_{1}} Q^{ \pm} R_{\alpha} \psi_{1}\right\|_{N F A\left[ \pm \kappa_{2}^{\prime}\right]^{*}} \lesssim\left|l-k_{3}\right| 2^{-\frac{l-k_{3}}{2}}\left\|P_{k_{1}} \psi_{1}\right\|_{S\left[k_{1}\right]}
$$

Hence we get

$$
\begin{aligned}
& \sum \\
& \kappa_{1,2}^{\prime} \in K_{\frac{l-k_{3}}{2}} \mid \operatorname{dist}\left( \pm \kappa_{1}^{\prime}, \pm \kappa_{2}^{\prime}\right) \sim 2 \frac{l-k_{3}}{2} \\
& 2^{\frac{l}{2}}\left\|P_{k_{1}, \kappa_{1}^{\prime}} Q^{ \pm} R_{\alpha} \psi_{1}\right\|_{N F A\left[ \pm \kappa_{2}^{\prime}\right]}\left\|\nabla^{-1} P_{k_{3}, \kappa_{2}^{\prime}} P_{k_{3}, \kappa_{3}} Q_{<k_{4}+20}^{ \pm} \psi_{3}\right\|_{P W\left[ \pm \kappa_{3}\right]} \\
& \lesssim 2^{\frac{k_{4}-k_{3}}{2}} 2^{\frac{l-k_{3}}{2}} 2^{-\frac{l-k_{3}}{2}}\left|k_{3}-l\right||| P_{k_{1}} \psi_{1}\left\|_{S\left[k_{1}\right]}|| P_{k_{3}, \kappa_{3}} Q_{<k_{4}+20} \psi_{3}\right\|_{S\left[k_{3}\right]}
\end{aligned}
$$

Note that since $k_{4}-k_{3}<<l-k_{3}$, the factor $\left|l-k_{3}\right|$ can be absorbed by $2^{\frac{k_{4}-k_{3}}{2}}$. Finally, we can wrap up case (iv) under the assumption $l>k_{4}+30$ : we have

$$
\begin{aligned}
& \sum_{\kappa_{2,3} \in K_{k_{4}-k_{2}} \mid \operatorname{dist}\left(\kappa_{2},-\kappa_{3}\right) \lesssim 2^{k_{4}-k_{2}}} \\
& \| P_{0}\left[\square P_{k_{1}+O(1)} Q_{l}\left[P_{k_{1}} Q_{<k_{4}-10} R_{\alpha} \psi_{1} \nabla^{-1} P_{k_{3}, \kappa_{3}} Q_{<k_{4}+20} \psi_{3}(.-y)\right]\right. \\
& \left.\nabla^{-1} P_{k_{2}, \kappa_{2}} Q_{<k_{4}+20} \psi_{2}(.-y)\right]\left.\right|_{N[0]} \\
& \lesssim 2^{\delta\left[l-k_{3}\right]} \sum_{\kappa_{1,2} \in K_{k_{4}-k_{2}} \mid \operatorname{dist}\left(\kappa_{2},-\kappa_{3}\right) \lesssim 2^{k_{4}-k_{2}}} \\
& \left\|\square P_{k_{1}+O(1)} Q_{l}\left[P_{k_{1}} Q_{<k_{4}-10} R_{\alpha} \psi_{1} \nabla^{-1} P_{k_{3}, \kappa_{3}} Q_{<k_{4}+20} \psi_{3}(.-y)\right]\right\|_{\dot{X}_{k_{1}}^{\frac{1}{2},-\frac{1}{2}, 1}} \\
& \left\|P_{k_{2}, \kappa_{2}} Q_{<k_{4}+20} \psi_{2}\right\|_{S\left[k_{2}\right]}
\end{aligned}
$$

By the preceding, we can estimate this by

$$
\begin{aligned}
& \sum_{\kappa_{2,3} \in K_{k_{4}-k_{2}} \mid \operatorname{dist}\left(\kappa_{2},-\kappa_{3}\right) \lesssim 2^{k_{4}-k_{2}}} \\
& 2^{\frac{k_{4}-k_{3}}{2}}\left|k_{3}-l\left\||| P_{k_{1}} \psi_{1}\right\|_{S\left[k_{1}\right]}\left\|P_{k_{2}, \kappa_{2}} Q_{<k_{4}+20} \psi_{2}\right\|_{S\left[k_{3}\right]}\left\|P_{k_{3}, \kappa_{3}} Q_{<k_{4}+20} \psi_{3}\right\|_{S\left[k_{3}\right]}\right.
\end{aligned}
$$

Using Cauchy-Schwartz as well as the observation from further above, we obtain the desired estimate by summing over $k_{4}+30 \leq l<k_{3}+O(1)$. 
Next, assume $l<k_{4}+30$. Here we decompose

$$
\begin{aligned}
& P_{k_{1}+O(1)} Q_{l}\left[P_{k_{1}} Q_{<k_{4}-10} R_{\alpha} \psi_{1} \nabla^{-1} P_{k_{3}, \kappa_{3}} Q_{<k_{4}+20} \psi_{3}(.-y)\right] \\
& =P_{k_{1}+O(1)} Q_{l}\left[P_{k_{1}} Q_{<k_{4}-10} R_{\alpha} \psi_{1} \nabla^{-1} P_{k_{3}, \kappa_{3}} Q_{l-10 \leq .<k_{4}+20} \psi_{3}(.-y)\right] \\
& +P_{k_{1}+O(1)} Q_{l}\left[P_{k_{1}} Q_{l-10 \leq .<k_{4}-10} R_{\alpha} \psi_{1} \nabla^{-1} P_{k_{3}, \kappa_{3}} Q_{<l-10} \psi_{3}(.-y)\right] \\
& +P_{k_{1}+O(1)} Q_{l}\left[P_{k_{1}} Q_{<l-10} R_{\alpha} \psi_{1} \nabla^{-1} P_{k_{3}, \kappa_{3}} Q_{<l-10} \psi_{3}(.-y)\right]
\end{aligned}
$$

For the first term, estimate

$$
\begin{aligned}
& \left\|\square P_{k_{1}+O(1)} Q_{l}\left[P_{k_{1}} Q_{<k_{4}-10} R_{\alpha} \psi_{1} \nabla^{-1} P_{k_{3}, \kappa_{3}} Q_{l-10 \leq .<k_{4}+20} \psi_{3}(.-y)\right]\right\|_{\dot{X}^{\frac{1}{2},-\frac{1}{2}, \infty}} \\
& \lesssim 2^{\frac{l}{2}} \sum_{j \in\left[l-10, k_{4}+20\right]}\left\|P_{k_{1}} Q_{<k_{4}-10} R_{\alpha} \psi_{1}\right\|_{L_{t}^{\infty} L_{x}^{2}} 2^{\frac{3 k_{3}}{2}} 2^{\frac{j-k_{3}}{2+}}\left\|\nabla^{-1} P_{k_{3}, \kappa_{3}} Q_{j} \psi_{3}\right\|_{L_{t}^{2} L_{x}^{2}}
\end{aligned}
$$

We have used here the 'improved Bernstein's inequality', see e. g. [24], [10]. This in turn we can bound by $\lesssim \sum_{j \in\left[l-10, k_{4}-10\right]} 2^{\frac{l-j}{2}} 2^{\frac{j-k_{3}}{2+}} \prod_{i=1,2}\left\|P_{k_{i}} \psi_{i}\right\|_{S\left[k_{i}\right]}$. Summing over $j$ and then over $l<k_{4}+20$, one easily obtains the gain $2^{\frac{k_{4}-k_{2}}{2+}}$, and from here the argument proceeds just as before. Next, we have

$$
\begin{aligned}
& P_{k_{1}+O(1)} Q_{l}\left[P_{k_{1}} Q_{l-10 \leq .<k_{4}-10} R_{\alpha} \psi_{1} \nabla^{-1} P_{k_{3}, \kappa_{3}} Q_{<l-10} \psi_{3}(.-y)\right] \\
& =\sum_{j \in\left[l-10, k_{4}-10\right]} P_{k_{1}+O(1)} Q_{l}\left[P_{k_{1}} Q_{j} R_{\alpha} \psi_{1} \nabla^{-1} P_{k_{3}, \kappa_{3}} Q_{<l-10} \psi_{3}(.-y)\right]
\end{aligned}
$$

Now we can simultaneously localize both $P_{k_{1}} Q_{j} R_{\alpha} \psi_{1}, \nabla^{-1} P_{k_{3}, \kappa_{3}} Q_{<l-10} \psi_{3}(.-y)$ to caps $\kappa_{1,2}^{\prime}$ of size $\sim 2^{\frac{j-k_{3}}{2}}$, such that $\pm \kappa_{1}^{\prime}, \pm \kappa_{2}^{\prime}$ have angular separation $\lesssim 2^{\frac{j-k_{3}}{2}}$. Hence we can write

$$
\begin{aligned}
& P_{k_{1}+O(1)} Q_{l}\left[P_{k_{1}} Q_{j} R_{\alpha} \psi_{1} \nabla^{-1} P_{k_{3}, \kappa_{3}} Q_{<l-10} \psi_{3}(.-y)\right] \\
& =\sum_{ \pm, \pm} \sum_{\kappa_{1,2}^{\prime} \in K_{\frac{j-k_{3}}{2}} \mid \operatorname{dist}\left( \pm \kappa_{1}^{\prime}, \pm \kappa_{2}^{\prime}\right) \leq 2^{\frac{j-k_{3}}{2}}} \\
& P_{k_{1}+O(1)} Q_{l}\left[P_{k_{1}, \kappa_{1}^{\prime}} Q_{j}^{ \pm} R_{\alpha} \psi_{1} \nabla^{-1} P_{k_{3}, \kappa_{2}^{\prime}} P_{k_{3}, \kappa_{3}} Q_{<l-10}^{ \pm} \psi_{3}(.-y)\right]
\end{aligned}
$$

Of course for fixed $\kappa_{1,2}^{\prime}$, there are only finitely many $\kappa_{3}$ for which this expression does not vanish. Then we can estimate

$$
\begin{aligned}
& \left\|\square P_{k_{1}+O(1)} Q_{l}\left[P_{k_{1}, \kappa_{1}^{\prime}} Q_{j}^{ \pm} R_{\alpha} \psi_{1} \nabla^{-1} P_{k_{3}, \kappa_{2}^{\prime}} P_{k_{3}, \kappa_{3}} Q_{<l-10}^{ \pm} \psi_{3}(.-y)\right]\right\|_{\dot{X}_{k_{1}}^{\frac{1}{2},-\frac{1}{2}, \infty}} \\
& \lesssim 2^{\frac{l}{2}}\left\|P_{k_{1}, \kappa_{1}^{\prime}} Q_{j}^{ \pm} R_{\alpha} \psi_{1}\right\|_{L_{t}^{2} L_{x}^{2}}\left\|\nabla^{-1} P_{k_{3}, \kappa_{2}^{\prime}} P_{k_{3}, \kappa_{3}} Q_{<l-10}^{ \pm} \psi_{3}\right\|_{L_{t}^{\infty} L_{x}^{\infty}}
\end{aligned}
$$

Using Bernstein's inequality for the 2nd factor, we estimate this by

$$
\lesssim 2^{\frac{l-j}{2}} 2^{\frac{j-k_{3}}{2}}|l-j| \prod_{i=1,2}\left\|P_{k_{1}, \kappa_{1}^{\prime}} \psi_{1}\right\|_{S\left[k_{1}\right]}\left\|P_{k_{3}, \kappa_{2}^{\prime}} \psi_{3}\right\|_{S\left[k_{3}\right]}
$$

Now, for fixed $j, l$, one sums over $\kappa_{1,2}^{\prime}$ as well as $\kappa_{2,3}$ (of which there are only finitely many for fixed $\kappa_{1,2}^{\prime}$ ), and finally sums over $j, l$ in the appropriate ranges, to obtain the desired estimate, just as in the case $l \geq k_{4}+30$.

The expression

$$
P_{k_{1}+O(1)} Q_{l}\left[P_{k_{1}} Q_{<l-10} R_{\alpha} \psi_{1} \nabla^{-1} P_{k_{3}, \kappa_{3}} Q_{<l-10} \psi_{3}(.-y)\right]
$$

is handled similarly. Here one localizes the inputs to caps $\kappa_{1,2}^{\prime}$ which are separated, and reasons as in the case $l \geq k_{4}$. 
This concludes case (iv).

(v): $\quad P_{k_{4}}\left(\nabla^{-1} P_{k_{2}} Q_{<k_{4}+20} \psi_{2} \square\left[\nabla^{-1} P_{k_{3}} Q_{<k_{4}+20} \psi_{3}\right] P_{k_{1}} R_{\alpha} Q_{<k_{4}-10} \psi_{1}\right.$. This is much simpler to estimate: use

$$
\begin{aligned}
& \| P_{k_{4}}\left(\nabla^{-1} P_{k_{2}} Q_{<k_{4}+20} \psi_{2} \square\left[\nabla^{-1} P_{k_{3}} Q_{<k_{4}+20} \psi_{3}\right] P_{k_{1}} R_{\alpha} Q_{<k_{4}-10} \psi_{1} \|_{L_{t}^{1} L_{x}^{2}}\right. \\
& \lesssim\left\|\square\left[\nabla^{-1} P_{k_{3}} Q_{<k_{4}+20} \psi_{3}\right]\right\|_{L_{t}^{2} L_{x}^{2}}\left\|\nabla^{-1} P_{k_{2}} Q_{<k_{4}+20} \psi_{2}\right\|_{L_{t}^{4} L_{x}^{\infty}}\left\|P_{k_{1}} R_{\alpha} Q_{<k_{4}-10} \psi_{1}\right\|_{L_{t}^{4} L_{x}^{\infty}}
\end{aligned}
$$

This can be estimated by $2^{\frac{k_{4}}{2}-\frac{k_{2}}{4}} \prod_{i=1}^{3}\left\|P_{k_{i}} \psi_{i}\right\|_{S\left[k_{i}\right]}$, which is better than what we need.

(vi): This is the expression

$$
\nabla^{-1} P_{k_{2}} Q_{<k_{4}+20} \psi_{2} \nabla^{-1} P_{k_{3}} Q_{<k_{4}+20} \psi_{3} \square P_{k_{1}} R_{\alpha} Q_{<k_{4}-10} \psi_{1}
$$

This is again straightforward: we estimate it by

$$
\begin{aligned}
& \left\|\nabla^{-1} P_{k_{2}} Q_{<k_{4}+20} \psi_{2} \nabla^{-1} P_{k_{3}} Q_{<k_{4}+20} \psi_{3} \square P_{k_{1}} R_{\alpha} Q_{<k_{4}-10} \psi_{1}\right\|_{L_{t}^{1} L_{x}^{2}} \\
& \lesssim\left\|\nabla^{-1} P_{k_{2}} Q_{<k_{4}+20} \psi_{2}\right\|_{L_{t}^{4} L_{x}^{\infty}}\left\|\nabla^{-1} P_{k_{3}} Q_{<k_{4}+20} \psi_{3}\right\|_{L_{t}^{4} L_{x}^{\infty}}\left\|\square P_{k_{1}} R_{\alpha} Q_{<k_{4}-10} \psi_{1}\right\|_{L_{t}^{2} L_{x}^{2}},
\end{aligned}
$$

and the last expression is easily estimated by $\lesssim 2^{\frac{k_{4}-k_{2}}{2}} \prod_{i=1}^{3}\left\|P_{k_{i}} \psi_{i}\right\|_{S\left[k_{i}\right]}$.

Now, we assume $k_{2}>20$. Here cases (i), (ii), (iii), (v), (vi) are handled in identical fashion. For (iv), we now have the identity

$$
\begin{aligned}
& \left.\left[P_{k_{1}} Q_{k_{4}-10} R_{\alpha} \psi_{1} P_{k_{3}} Q_{<k_{4}+20} \psi_{3}(.-y)\right]\right] \\
& \left.=P_{k_{3}+O(1)} Q_{<k_{1}+O(1)}\left[P_{k_{1}} Q_{k_{4}-10} R_{\alpha} \psi_{1} P_{k_{3}, \kappa_{3}} Q_{<k_{4}+20} \nabla^{-1} \psi_{3}(.-y)\right]\right]
\end{aligned}
$$

Freeze the modulation to size $2^{l}$. Then we need to distinguish between the cases $l \geq k_{4}, l<k_{4}$. The calculations are essentially the same, so we outline how to deal wit the first case. Now one writes

$$
\begin{aligned}
& \left.P_{k_{3}+O(1)} Q_{l}\left[P_{k_{1}} Q_{k_{4}-10} R_{\alpha} \psi_{1} P_{k_{3}} Q_{<k_{4}+20} \nabla^{-1} \psi_{3}(.-y)\right]\right] \\
& =\sum_{\kappa_{1,2}^{\prime} \in K_{\frac{l-k_{1}}{2}} \mid \operatorname{dist}\left( \pm \kappa_{1}^{\prime}, \pm \kappa_{2}^{\prime}\right) \sim 2}^{\frac{l-k_{1}}{2}} \\
& \left.P_{k_{3}+O(1)} Q_{l}\left[P_{k_{1}, \kappa_{1}^{\prime}} Q_{k_{4}-10}^{ \pm} R_{\alpha} \psi_{1} P_{k_{3}, \kappa_{2}^{\prime}} P_{k_{3}, \kappa_{3}} Q_{<k_{4}+20}^{ \pm} \nabla^{-1} \psi_{3}(.-y)\right]\right]
\end{aligned}
$$

From this point on the proof is an exact replica of the case $k_{3}<-20$.

The case $k_{2} \in[-20,20]$ is also essentially the same, the only difference being that now in case (iv), the term $\left.\left[P_{k_{1}} Q_{<k_{4}-10} R_{\alpha} \psi_{1} P_{k_{3}} Q_{<k_{4}+20} \nabla^{-1} \psi_{3}(.-y)\right]\right]$ may have very small frequency. This is handled similarly, see e. g. [11], where such an argument is carried out in detail. 
(4) The 2nd null-form. This is handled just like the first null-form, the only difference being that one uses the identity

$$
\begin{aligned}
& \sum_{j=1}^{3} \triangle^{-1} \partial_{j} \partial^{\nu}\left[R_{\nu} f R_{j} g-R_{j} f R_{\nu} g\right] h \\
& =\square\left[\sum_{j=1}^{3} \triangle^{-1} \partial_{j}\left(\nabla^{-1} f R_{j} g\right)\right] h-\frac{1}{2} \square\left(\nabla^{-1} f\right) \nabla^{-1} g h \\
& -\frac{1}{2} \square\left[\nabla^{-1} f \nabla^{-1} g\right] h+\frac{1}{2} \square\left(\nabla^{-1} g\right) \nabla^{-1} f h
\end{aligned}
$$

These can be handled just as the terms in the identity underlying (3).

The remaining expressions in the statement of Proposition 4.6 can be estimated in the same way.

We next state the following Proposition needed to handle the quintilinear and higher order terms:

Proposition 4.9. We have the bounds

$$
\begin{aligned}
& \left\|P_{k} \nabla_{x, t}\left[P_{k_{1}} \psi_{1} P_{r_{1}} \nabla^{-1}\left[P_{r_{2}} \nabla^{-1}\left[P_{k_{2}} \psi_{2} \nabla^{-1} P_{r_{3}}\left[P_{k_{3}} \psi_{3} P_{k_{4}} \psi_{4}\right]\right] P_{k_{5}} R_{\nu} \psi_{5}\right]\right]\right\|_{N[k]} \\
& \lesssim 2^{-\delta\left[\left|k-k_{1}\right|+\left|k_{3}-k_{4}\right|\right]} 2^{\delta\left[\min \left\{k_{2,5}, r_{1,2,3}\right\}-\max \left\{k_{2,5}, r_{1,2,3}\right\}\right]} \prod_{i=1}^{5}\left\|P_{k_{i}} \psi_{i}\right\|_{S\left[k_{i}\right]}
\end{aligned}
$$

for suitable $\delta>0$. Analogous bounds hold for the other higher order terms in (2.3):

$$
\begin{aligned}
& \left\|P_{k} \nabla_{x, t}\left[P_{r_{1}} \nabla^{-1}\left[P_{k_{1}} \psi_{1} P_{r_{2}} \nabla^{-1}\left[P_{k_{2}} \psi_{2} P_{k_{3}} \psi_{3}\right]\right] P_{r_{3}} \nabla^{-1}\left[P_{k_{4}} \psi_{4} P_{k_{5}} \psi_{5}\right]\right]\right\|_{N[k]} \\
& \lesssim 2^{-\delta\left[\left|k_{4}-k_{5}\right|+\left|k_{2}-k_{3}\right|\right]} 2^{\delta\left[\min \left\{r_{1,3}, k\right\}-\max \left\{r_{1,3}, k\right\}\right]} 2^{\delta\left[\min \left\{r_{1,2}, k_{1}\right\}-\max \left\{r_{1,2}, k_{1}\right\}\right]} \\
& \prod_{i=1}^{5}\left\|P_{k_{i}} \psi_{i}\right\|_{S\left[k_{i}\right]}
\end{aligned}
$$

Proof. We show the first estimate, the 2 nd following in a similar vein. This is in principle straightforward given Lemma 4.1, the only difficulty being the operator $\nabla_{x, t}$ in front. To deal with it, assume the output is at frequency $\sim 1$ ( as we may by scale invariance) as well as modulation $2^{l}, l>>1$. Thus this is the expression

$$
P_{0} Q_{l} \nabla_{x, t}\left[P_{k_{1}} \psi_{1} P_{r_{1}} \nabla^{-1}\left[P_{r_{2}} \nabla^{-1}\left[P_{k_{2}} \psi_{2} \nabla^{-1} P_{r_{3}}\left[P_{k_{3}} \psi_{3} P_{k_{4}} \psi_{4}\right]\right] P_{k_{5}} R_{\nu} \psi_{5}\right]\right]
$$

Then either $k_{1} \geq l-10$, or $P_{k_{1}} \psi_{1}$ has modulation $\geq 2^{l-10}$, or else $P_{r_{1}} \nabla^{-1} \ldots$ has modulation $\geq 2^{l-10}$. In the last case, repeat the same argument with

$$
P_{r_{1}} \nabla^{-1}\left[P_{r_{2}} \nabla^{-1}\left[P_{k_{2}} \psi_{2} \nabla^{-1} P_{r_{3}}\left[P_{k_{3}} \psi_{3} P_{k_{4}} \psi_{4}\right]\right] P_{k_{5}} R_{\nu} \psi_{5}\right]
$$

The conclusion is that at least one of the inputs needs to have frequency at least comparable to $2^{l}$, or else at least one input needs to have modulation at least comparable to $2^{l}$. Note that we can organize the expression as

$$
\nabla_{x, t} P_{0} Q_{l}\left[P_{k_{1}} \psi_{1} P_{r_{1}} \nabla^{-1}\left[X P_{k_{5}} R_{\nu} \psi_{5}\right]\right]
$$

with

$$
X=P_{r_{2}} \nabla^{-1}\left[P_{k_{2}} \psi_{2} \nabla^{-1} P_{r_{3}}\left[P_{k_{3}} \psi_{3} P_{k_{4}} \psi_{4}\right]\right]
$$


Then use the decomposition

$$
\begin{aligned}
& \nabla_{x, t} P_{0} Q_{l}\left[P_{k_{1}} \psi_{1} P_{r_{1}} \nabla^{-1}\left[X P_{k_{5}} R_{\nu} \psi_{5}\right]\right] \\
& =\chi_{k_{1} \geq l-100} \nabla_{x, t} P_{0} Q_{l}\left[P_{k_{1}} \psi_{1} P_{r_{1}} \nabla^{-1}\left[X P_{k_{5}} R_{\nu} \psi_{5}\right]\right] \\
& +\chi_{k_{1}<l-100} \nabla_{x, t} P_{0} Q_{l}\left[P_{k_{1}} Q_{\geq l-10} \psi_{1} P_{r_{1}} \nabla^{-1}\left[X P_{k_{5}} R_{\nu} \psi_{5}\right]\right] \\
& +\chi_{k_{1}<l-100} \nabla_{x, t} P_{0} Q_{l}\left[P_{k_{1}} Q_{<l-10} \psi_{1} P_{r_{1}} Q_{\geq l-10} \nabla^{-1}\left[X P_{k_{5}} R_{\nu} \psi_{5}\right]\right]
\end{aligned}
$$

To estimate the first and 2nd terms, we use the following

LEMma 4.10. We have the bounds

$$
\|X\|_{L_{t}^{2} \dot{H}_{x}^{1} \cap L_{t}^{2} \dot{W}_{x}^{\infty,-\frac{1}{2}} \cap L_{t}^{\infty} \dot{H}_{x}^{\frac{1}{2}}} \lesssim 2^{\delta\left[\min \left\{r_{2,3}, k_{2,3,4}\right\}-\max \left\{r_{2,3}, k_{2,3,4}\right\}\right]} \prod_{i=2,3,4}\left\|P_{k_{i}} \psi_{i}\right\|_{S\left[k_{i}\right]}
$$

Proof. (Lemma 4.10) simple application of Bernstein's inequality.

Then we compute (for suitable $\delta>0$ )

$$
\begin{aligned}
& \left\|\chi_{k_{1} \geq l-100} \nabla_{x, t} P_{0} Q_{l}\left[P_{k_{1}} \psi_{1} P_{r_{1}} \nabla^{-1}\left[X P_{k_{5}} R_{\nu} \psi_{5}\right]\right]\right\|_{\dot{X}_{0}^{-\frac{1}{2},-\frac{1}{2}, \infty}} \\
& \lesssim 2^{\frac{1}{2} l}\left\|P_{k_{1}} \psi_{1}\right\|_{L_{t}^{\infty} L_{x}^{2}}\left\|P_{k_{1}+O(1)} \nabla^{-1}\left[X P_{k_{5}} R_{\nu} \psi_{5}\right]\right\|_{L_{t}^{2} L_{x}^{2}} \\
& \lesssim 2^{\delta\left[\min \left\{r_{2,3}, k_{2,3,4}\right\}-\max \left\{r_{2,3}, k_{2,3,4}\right\}\right]} \prod_{i=2,3,4}\left\|P_{k_{i}} \psi_{i}\right\|_{S\left[k_{i}\right]} \\
& \times 2^{\frac{l}{2}} 2^{-k_{1}} 2^{\delta\left[\min \left\{k_{1}, r_{2}, k_{5}\right\}-\max \left\{k_{1}, r_{2}, k_{5}\right\}\right]} \prod_{i=1,5}\left\|P_{k_{i}} \psi_{i}\right\|_{S\left[k_{i}\right]}
\end{aligned}
$$

where we have again invoked Bernstein's inequality a number of times in the last step. This is of course more than enough to get the estimate of Proposition 4.9 in this case. Next, estimate

$$
\begin{aligned}
& \left\|\chi_{k_{1}<l-100} \nabla_{x, t} P_{0} Q_{l}\left[P_{k_{1}} Q_{\geq l-10} \psi_{1} P_{r_{1}} \nabla^{-1}\left[X P_{k_{5}} R_{\nu} \psi_{5}\right]\right]\right\|_{N[0]} \\
& \lesssim 2^{\frac{l}{2}} \min \left\{\left\|P_{k_{1}} Q_{\geq l-10} \psi_{1}\right\|_{L_{t}^{2} L_{x}^{2}} \|\left. P_{r_{1}} \nabla^{-1}\left[X P_{k_{5}} R_{\nu} \psi_{5}\right]\right|_{L_{t}^{\infty} L_{x}^{\infty}+L_{t}^{\infty} L_{x}^{2}},\right. \\
& \left.\left\|P_{k_{1}} Q_{\geq l-10} \psi_{1}\right\|_{L_{t}^{2} L_{x}^{\infty}} \mid P_{r_{1}} \nabla^{-1}\left[X P_{k_{5}} R_{\nu} \psi_{5}\right] \|_{L_{t}^{\infty} L_{x}^{2}}\right\}
\end{aligned}
$$

Again, one checks this is bounded by an expression as in the Proposition. Finally, for the last term, use a similar decomposition

$$
\begin{aligned}
& P_{r_{1}} Q_{\geq l-10} \nabla^{-1}\left[X P_{k_{5}} R_{\nu} \psi_{5}\right] \\
& =\chi_{k_{5} \geq l-100} P_{r_{1}} Q_{\geq l-10} \nabla^{-1}\left[X P_{k_{5}} R_{\nu} \psi_{5}\right] \\
& +\chi_{k_{5}<l-100} P_{r_{1}} Q_{\geq l-10} \nabla^{-1}\left[X P_{k_{5}} R_{\nu} Q_{\geq l-20} \psi_{5}\right] \\
& +\chi_{k_{5}<l-100} P_{r_{1}} Q_{\geq l-10} \nabla^{-1}\left[Q_{\geq l-20} X P_{k_{5}} R_{\nu} Q_{<l-20} \psi_{5}\right]
\end{aligned}
$$

For the first term here, use that

$$
\left\|\left.\chi_{k_{5} \geq l-100} P_{r_{1}} Q_{\geq l-10} \nabla^{-1}\left[X P_{k_{5}} R_{\nu} \psi_{5}\right]\right|_{L_{t}^{2} L_{x}^{2}} \lesssim 2^{\frac{r_{1}}{2}}|| X||_{L_{t}^{2} L_{x}^{2}}|| R_{\nu} \psi_{5}\right\|_{L_{t}^{\infty} L_{x}^{2}}
$$

and from here one concludes

$$
\begin{aligned}
& \left\|\chi_{k_{1}<l-100} \nabla_{x, t} P_{0} Q_{l}\left[P_{k_{1}} Q_{<l-10} \psi_{1} \chi_{k_{5} \geq l-100} P_{r_{1}} Q_{\geq l-10} \nabla^{-1}\left[X P_{k_{5}} R_{\nu} \psi_{5}\right]\right]\right\|_{N[0]} \\
& \lesssim 2^{\frac{l}{2}}\left\|P_{k_{1}} Q_{<l-10} \psi_{1}\right\|_{L_{t}^{\infty} L_{x}^{\infty}}\left\|P_{k_{5}+O(1)} X\right\|_{L_{t}^{2} L_{x}^{2}}\left\|P_{k_{5}} R_{\nu} \psi_{5}\right\|_{L_{t}^{\infty} L_{x}^{2}} \\
& \lesssim 2^{\frac{l}{2}+k_{1}-\frac{3}{2} k_{5}} 2^{\delta\left(\min \left\{r_{2,3}, k_{2,3,4}\right\}-\max \left\{r_{2,3}, k_{2,3,4}\right\}\right)} \prod_{i=i}^{5}\left\|P_{k_{i}} \psi_{i}\right\|_{S\left[k_{i}\right]}
\end{aligned}
$$


Further, write

$$
\begin{aligned}
& \chi_{k_{5}<l-100} P_{r_{1}} Q_{\geq l-10} \nabla^{-1}\left[X P_{k_{5}} R_{\nu} Q_{\geq l-20} \psi_{5}\right] \\
& =\sum_{l_{1} \geq l-20} \chi_{k_{5}<l-100} P_{r_{1}} Q_{\geq l-10} \nabla^{-1}\left[X P_{k_{5}} R_{\nu} Q_{l_{1}} \psi_{5}\right]
\end{aligned}
$$

Then we get

$$
\begin{aligned}
& \| \sum_{l_{1} \geq l-20} \chi_{k_{1}<l-100} \nabla_{x, t} P_{0} Q_{l}\left[P_{k_{1}} Q_{<l-10} \psi_{1} \chi_{k_{5}<l-100} P_{r_{1}} Q_{\geq l-10}\right. \\
& \left.\nabla^{-1}\left[X P_{k_{5}} Q_{l_{1}} R_{\nu} \psi_{5}\right]\right] \|_{N[0]} \\
& \lesssim \sum_{l_{1} \geq l-20} 2^{\frac{1}{2}\left(l-l_{1}\right)} 2^{\delta\left(\min \left\{k_{1,2,3,4,5}, r_{1,2,3}\right\}-\max \left\{k_{1,2,3,4,5}, r_{1,2,3}\right\}\right)} \prod_{i=1}^{5}\left\|P_{k_{i}} \psi_{i}\right\|_{S\left[k_{i}\right]}
\end{aligned}
$$

for suitable $\delta>0$. Finally, consider the contribution of

$$
\chi_{k_{5}<l-100} P_{r_{1}} Q_{\geq l-10} \nabla^{-1}\left[Q_{\geq l-20} X P_{k_{5}} R_{\nu} Q_{<l-20} \psi_{5}\right]
$$

Here we use

Lemma 4.11. The following estimate holds:

$$
\left\|Q_{l_{1}} X\right\|_{L_{t}^{2} L_{x}^{2}} \lesssim 2^{-\frac{1}{2} l_{1}} 2^{-\frac{1}{2} r_{2}} 2^{\delta\left(\min \left\{r_{2}, r_{3}, k_{2,3,4}\right\}-\max \left\{r_{2}, r_{3}, k_{2,3,4}\right\}\right)} \prod_{i=2,3,4}\left\|P_{k_{i}} \psi_{i}\right\|_{S\left[k_{i}\right]}
$$

for suitable $\delta>0$.

Proof. (Lemma 4.11) Use reasoning as in Lemma 4.8, as well as above.

Using this, we can estimate

$$
\begin{aligned}
& \| \chi_{k_{1}<l-100} \nabla_{x, t} P_{0} Q_{l}\left[P_{k_{1}} \psi_{1} \chi_{k_{5}<l-100} P_{r_{1}} Q_{\geq l-10} \nabla^{-1}\left[Q_{\geq l-20} X P_{k_{5}} R_{\nu} Q_{<l-20} \psi_{5}\right] \|_{N[0]}\right. \\
& \leq \sum_{l_{1} \geq l-20} 2^{\frac{l}{2}} \| \chi_{k_{1}<l-100} P_{0} Q_{l}\left[P_{k_{1}} \psi_{1} \chi_{k_{5}<l-100} P_{r_{1}} Q_{\geq l-10}\right. \\
& \nabla^{-1}\left[Q_{l_{1}} X P_{k_{5}} R_{\nu} Q_{<l-20} \psi_{5}\right] \|_{L_{t}^{2} L_{x}^{2}} \\
& \lesssim \sum_{l_{1} \geq l-20} 2^{\frac{1}{2}\left(l-l_{1}\right)} 2^{\delta\left[\min \left\{k_{1,2,3,4,5}, r_{1,2,3}\right\}-\max \left\{k_{1,2,3,4,5}, r_{1,2,3}\right\}\right]} \prod_{i=1}^{5}\left\|P_{k_{i}} \psi_{i}\right\|_{S\left[k_{i}\right]}
\end{aligned}
$$

for suitable $\delta>0$. This follows as usual via various frequency trichotomies as well as Bernstein's inequality. This concludes dealing with the large output-at-largemodulation-case, and we now need to focus on

$$
\left.\nabla_{x, t} P_{0} Q_{<20}\left[P_{k_{1}} \psi_{1} P_{r_{1}} \nabla^{-1}\left[P_{r_{2}} \nabla^{-1}\left[P_{k_{2}} \psi_{2} \nabla^{-1} P_{r_{3}}\left[P_{k_{3}} \psi_{3} P_{k_{4}} \psi_{4}\right]\right] P_{k_{5}} R_{\nu} \psi_{5}\right]\right]\right]
$$

This we do via the following

LEMMA 4.12. We have the estimate

$$
\begin{aligned}
& 2^{\left(\frac{3}{4}+\frac{3}{p}-1\right) r_{2}} \| P_{r_{2}} \nabla^{-1}\left[P _ { k _ { 2 } } \psi _ { 2 } \nabla ^ { - 1 } P _ { r _ { 3 } } \left[P_{k_{3}} \psi_{3} P_{k_{4}} \psi_{4} \|_{L_{t}^{\frac{4}{3}} L_{x}^{p}}\right.\right. \\
& \lesssim 2^{\delta\left[\min \left\{k_{2}, r_{2,3}\right\}-\max \left\{k_{2}, r_{2,3}\right\}\right]} 2^{-\delta\left|k_{3}-k_{4}\right|} \prod_{i=2,3,4}\left\|P_{k_{i}} \psi_{i}\right\|_{S\left[k_{i}\right]}
\end{aligned}
$$

for suitable $\delta>0$ and $p \geq 2$.

Proof. (Lemma 4.12) Follows from Lemma 4.2 and some simple frequency trichotomies and Bernstein's inequality. 
From here one easily concludes

$$
\begin{aligned}
& \left.\| \nabla_{x, t} P_{0} Q_{<20}\left[P_{k_{1}} \psi_{1} P_{r_{1}} \nabla^{-1}\left[P_{r_{2}} \nabla^{-1}\left[P_{k_{2}} \psi_{2} \nabla^{-1} P_{r_{3}}\left[P_{k_{3}} \psi_{3} P_{k_{4}} \psi_{4}\right]\right] P_{k_{5}} R_{\nu} \psi_{5}\right]\right]\right] \|_{L_{t}^{1} \dot{H}_{x}^{-\frac{1}{2}}} \\
& \lesssim 2^{-\delta\left[\left|k_{1}\right|+\left|k_{3}-k_{4}\right|\right]} 2^{\delta\left[\min \left\{k_{2,5}, r_{1,2,3}\right\}-\max \left\{k_{2,5}, r_{1,2,3}\right\}\right]} \prod_{i=1}^{5}\left\|P_{k_{i}} \psi_{i}\right\|_{S\left[k_{i}\right]},
\end{aligned}
$$

again choosing $\delta>0$ small enough. This concludes the proof of Proposition 4.9 for the first inequality. The 2 nd follows similar reasoning.

We now continue with the proof of Proposition 4.5, where we need to establish the last 3 inequalities for the iterates $\psi_{\alpha, 1, j}$. This only requires some additional work for the terms involving the vector fields $\Gamma^{\beta}$. To get estimates on $\Gamma^{\beta} \psi_{\alpha, 1, j}$, we need commute the vector fields $\Gamma^{\beta}$ inside the source terms on the right hand side of (2.6). To do this, we require the following simple

LEMma 4.13. The following commutator bounds obtain for any translation invariant norm $\|\cdot\|_{S}$ :

$$
\begin{gathered}
\left\|\left[\Gamma^{\beta}, \square^{-1} P_{k} Q_{\geq k}\right] \psi\right\|_{S} \lesssim 2^{-2 k} 2^{-|\beta| k}|| \nabla_{x, t}^{|\beta|} P_{k} \psi \|_{S}+(|\beta|-1) \\
\sum_{|\gamma|=|\beta|-1} 2^{-3 k}|| \nabla_{x, t} \Gamma^{\gamma} P_{k} \psi \|_{S} \\
\left\|\left[\Gamma^{\beta}, P_{k} Q_{<k+\delta l}\right] \psi\right\|\left\|_{S} \lesssim 2^{-|\beta| k}|| \nabla_{x, t}^{|\beta|} P_{k} \psi\right\|_{S}+(|\beta|-1) \sum_{|\gamma|=|\beta|-1} 2^{-k}\left\|\nabla_{x, t} \Gamma^{\gamma} P_{k} \psi\right\| \|_{S}
\end{gathered}
$$

First considering the case $|\beta|=1$, note that $\square^{-1} P_{k} Q_{\geq k}$ is given by convolution with a function $a(t, x)$ with the property $\|a(t, x)\|_{L_{t, x}^{1}\left(\mathbb{R}^{3+1}\right.} \lesssim 2^{-2 k}$. Then we have

$$
\begin{aligned}
& {\left[\Gamma, \square^{-1} P_{k} Q_{\geq k}\right]=\Gamma_{t, x} \int_{\mathbb{R}^{3+1}} a(t-s, x-y) \psi(s, y) d s d y} \\
& -\int_{\mathbb{R}^{3+1}} a(t-s, x-y) \Gamma_{s, y} \psi(s, y) d s d y
\end{aligned}
$$

Taking into account that $\Gamma$ is of the form $\partial_{t}, \partial_{x_{i}}, x_{i} \partial_{x_{j}}-x_{j} \partial_{x_{i}}, t \partial_{t}+\sum_{i=1}^{3} x_{i} \partial_{x_{i}}, x_{i} \partial_{t}+$ $t \partial_{x_{i}}$, we see the above integral can be written in the form

$$
\int_{\mathbb{R}^{3+1}} s a(s, y) \nabla_{t, x} \psi(t-s, x-y) d s d y+\int_{\mathbb{R}^{3+1}} y a(s, y) \nabla_{t, x} \psi(t-s, x-y) d s d y
$$

Then conclude by observing

$$
\|s a(s, y)\|_{L_{s, y}^{1}}+\||| y \mid(s, y)\|_{L_{s, y}^{1}} \lesssim 2^{-3 k}
$$

For higher order operators, use

$$
\left.\left[\Gamma_{1} \Gamma_{2}, \square^{-1} P_{k} Q_{\geq k}\right]=\Gamma_{1}\left[\Gamma_{2}, \square^{-1} P_{k} Q_{\geq k}\right]+\left[\Gamma_{1}, \square^{-1} P_{k} Q_{\geq k}\right]\right] \Gamma_{2},
$$

as well as the fact that $\left[\Gamma_{2}, \square^{-1} P_{k} Q_{\geq k}\right]$ can be written as a convolution operator whose kernel enjoys similar properties to the one associated with $\square^{-1} P_{k} Q_{\geq k}$. The proof or $\left[\Gamma^{\beta}, P_{k} Q_{<k+\delta l}\right]$ is similar.

With this in hand, observe that we have

$$
\begin{aligned}
& \square\left[\Gamma \psi_{\alpha, 1, j+1}\right]=[\square, \Gamma] \psi_{\alpha, 1, j+1}+\Gamma \square\left[\eta_{T}(t) S(t)\left(\psi_{\alpha}[0]\right)\right] \\
& +\Gamma \square\left[\int_{0}^{t} \eta_{T}(t-s) \mathbf{P}_{[-\delta l, \delta l]} F\left(\psi_{1, j}\right)(s) d s\right]
\end{aligned}
$$


Then clearly we have

$$
\left.\square\left[\Gamma \psi_{\alpha, 1, j+1}\right]\right|_{[-T, T]}=\left.[\square, \Gamma] \psi_{\alpha, 1, j+1}\right|_{[-T, T]}+\left.\Gamma \mathbf{P}_{[-\delta l, \delta l]} F\left(\psi_{1, j}\right)\right|_{[-T, T]}
$$

In order to estimate the right hand side, and in particular the right most term, observe that application of $\Gamma$ does leave the null-structure (4.1) intact: indeed, $[\square, \Gamma]=c \square$ for each vector field $\Gamma$ and a suitable number $c=c_{\Gamma}$. Re-iterating the proofs of Proposition 4.6, 4.9, and applying the preceding Lemma, we can conclude that

$$
\left\|\left.\Gamma \mathbf{P}_{[-\delta l, \delta l]} F\left(\psi_{1, j}\right)\right|_{[-T, T]}\right\|_{N[k]\left([-T, T] \times \mathbb{R}^{3}\right)} \lesssim \epsilon \tilde{M} 2^{C \delta l} c_{k},
$$

provided we make the bootstrap assumption $\left\|P_{k} \Gamma \psi_{1}\right\|_{S[k]\left([-T, T] \times \mathbb{R}^{3}\right)} \lesssim \tilde{M} 2^{C \delta l} c_{k}$. The claim of the Proposition follows when $|\beta|=1$ for $\psi_{\alpha, 1, j+1}$, by using the energy inequality Theorem 4.4, and one argues similarly for $\Gamma^{\beta} \psi_{\alpha, 1},|\beta|=2$, etc. This concludes the proof of Proposition 4.5.

Next, we explain the proof of Proposition 2.2:

Proof. (Proposition 2.2) We shall again use Proposition 4.6, Proposition 4.9, as well as some elementary additional estimates. Fix a time scale $l>>1$ and consider $\mathrm{t} \psi_{\alpha, 2}$ defined on $\left[-2^{-l+2}, 2^{l+2}\right]$ as in the preceding. The equations satisfied by the $\psi_{\alpha, 2}=\psi_{\alpha}-\psi_{\alpha, 1}$ is as follows:

$$
\square \psi_{\alpha, 2}=F\left(\psi_{1}, \psi_{2}\right)+\left(1-\mathbf{P}_{[-\delta l, \delta l]}\right) F\left(\psi_{1}\right), \psi_{\alpha, 2}
$$

Here $F\left(\psi_{1}, \psi_{2}\right)$ is the right hand side of (2.3) but with $\psi$ replaced by $\psi_{1,2}$, and the additional requirement that at least one copy of $\psi_{2}$ is present. We need to establish the following

Bootstrapping step: Let $0 \leq T \leq 2^{l+2}$ and assume that we have

$$
\left\|P_{k} \psi_{\alpha, 2}\right\|_{S[k]\left([-T, T] \times \mathbb{R}^{3}\right)} \leq M e_{k}, \alpha=0,1,2,3
$$

for some $M$ larger than some $M_{0}=M_{0}(\psi[0])$. Then we have

$$
\left\|P_{k} \psi_{\alpha, 2}\right\|_{S[k]\left([-T, T] \times \mathbb{R}^{3}\right)} \leq \frac{M}{2} e_{k}, \alpha=0,1,2,3
$$

We recall the definition of $e_{k}:=\min \left\{d_{k}, 2^{\mu(\max \{k-\delta l,-k-\delta l\})} d_{k}\right\}$ for $\mu>0$ sufficiently small. Having established the bootstrapping, Proposition 2.2 will then follow from the fact that (see e. g. [11])

$$
\lim _{T \rightarrow 0} \sup _{k \in \mathbb{Z}}\left\|P_{k} \psi_{\alpha, 2}\right\|_{S[k]\left([-T, T] \times \mathbb{R}^{3}\right)}=0
$$

We will show that the assumption above implies

$$
\left\|P_{k} F\left(\psi_{1}, \psi_{2}\right)\right\|_{N[k]\left([-T, T] \times \mathbb{R}^{3}\right)}+\left\|P_{k}\left(1-\mathbf{P}_{[-\delta l, \delta l]}\right) F\left(\psi_{1}\right)\right\|_{N[k]\left([-T, T] \times \mathbb{R}^{3}\right)} \lesssim \epsilon M^{9} e_{k},
$$

which implies the bootstrapping step by invoking Theorem 4.4. We shall first prove the estimate

$$
\left\|P_{k} F\left(\psi_{1}, \psi_{2}\right)\right\|_{N[k]\left([-T, T] \times \mathbb{R}^{3}\right)} \lesssim \epsilon M^{9} e_{k}
$$


Recall that $P_{k} F\left(\psi_{1}, \psi_{2}\right)$ consists of trilinear null-forms as well as quintilinear and higher order terms. From Proposition 4.6, we have the schematic relation

$$
\begin{aligned}
& \left\|" P_{k} \nabla_{x, t}\left[P_{k_{1}} \psi_{1} \nabla^{-1} P_{k_{4}}\left[P_{k_{2}} \psi_{2} P_{k_{3}} \psi_{3}\right]\right] "\right\|_{N[k]\left([-T, T] \times \mathbb{R}^{3}\right)} \\
& \lesssim 2^{-\delta_{1}\left|k_{2}-k_{3}\right|} 2^{-\delta_{2}\left[k_{4}-\max \left\{k_{2}, k_{3}\right\}\right]} 2^{-\delta_{3}\left|k-k_{1}\right|} \prod_{i=1}^{3}\left\|P_{k_{i}} \psi_{i}\right\|_{S\left[k_{i}\right]\left([-T, T] \times \mathbb{R}^{3}\right)}
\end{aligned}
$$

Here the term " $P_{k} \nabla_{x, t} \ldots$ " of course stands for one of the trilinear null-forms in (2.3). Now, we we think of the entries $\psi_{1}, \psi_{2}, \psi_{3}$ as either given by a $\psi_{\alpha, 1}$ or a $\psi_{\alpha, 2}$, with at least one occurence of $\psi_{\alpha, 2}$. Then we have two possible cases:

(i): at least one copy of $\psi_{\alpha, 2}$ inside $\nabla^{-1} P_{k_{4}}[\ldots]$. Let this be $P_{k_{2}} \psi_{\alpha, 2}$.

This we estimate by

$$
\lesssim 2^{-\delta_{3}\left|k-k_{1}\right|} 2^{-\delta_{1}\left|k_{2}-k_{3}\right|} 2^{-\delta_{2}\left|k_{4}-\max \left\{k_{2}, k_{3}\right\}\right|} d_{k_{1}} e_{k_{2}} d_{k_{3}}
$$

Now use that

$$
e_{k_{2}}=\min \left\{d_{k_{2}}, 2^{\mu \max \left\{k_{2}-\delta l,-k_{2}-\delta l\right\}} d_{k_{2}}\right\} \lesssim 2^{-\mu \delta l}
$$

since we have $d_{k} \lesssim 2^{-\sigma|k|}$ for some $\sigma>0$, and we assume that $\mu$ is chosen small enough. Then we have

$$
\sum_{k_{1} \in \mathbb{Z}} 2^{-\mu \delta l} 2^{-\delta_{3}\left|k-k_{1}\right|} d_{k_{1}} \lesssim e_{k}
$$

We can now infer that

$$
\sum_{k_{1,2,3} \in \mathbb{Z}} 2^{-\delta_{3}\left|k-k_{1}\right|} 2^{-\delta_{1}\left|k_{2}-k_{3}\right|} 2^{-\delta_{2}\left|k_{4}-\max \left\{k_{2}, k_{3}\right\}\right|} d_{k_{1}} e_{k_{2}} d_{k_{3}} \lesssim \epsilon e_{k}
$$

(ii): The first input $P_{k_{1}} \psi_{1}$ is $P_{k_{1}} \psi_{\alpha, 2}$. Here we infer the desired bound directly since the $e_{k}$ form a frequency envelope.

The remaining terms in $F\left(\psi_{1}, \psi_{2}\right)$ if higher degree of multilinearity are treated similarly and omitted here.

Now we turn to estimating the term

$$
P_{k}\left(1-\mathbf{P}_{[-\delta l, \delta l]}\right) F\left(\psi_{1}\right)
$$

Recalling the definition of $-\mathbf{P}_{[-\delta l, \delta l]}$, we see that in the expressions constituting $\left(1-\mathbf{P}_{[-\delta l, \delta l]}\right) F\left(\psi_{1}\right)$ either at least one frequency $2^{k}$ satisfies $|k| \geq \delta l$, or else at least one operator $P_{k} Q_{\geq k+\delta l}$ occurs. We need to show that this implies an extra exponential gain in $l$. We do this here for the trilinear terms, the higher order terms being treated similarly: first, again using the schematic notation

$$
" P_{k} \nabla_{x, t}\left[P_{k_{1}} \psi_{1} \nabla^{-1} P_{k_{4}}\left[P_{k_{2}} \psi_{2} P_{k_{3}} \psi_{3}\right]\right] ",
$$

assume that one of $k_{1,2,3,4}$ is of large absolute size. Then reasoning exactly as before in (i), (ii), implies

$$
\left\|" P_{k} \nabla_{x, t}\left[P_{k_{1}} \psi_{1} \nabla^{-1} P_{k_{4}}\left[P_{k_{2}} \psi_{2} P_{k_{3}} \psi_{3}\right]\right] "\right\|_{N[k]} \lesssim e_{k}
$$

Further, we have 
LEMMA 4.14. The following estimates hold, where we again use schematic notation:

$$
\begin{aligned}
& \left\|" P_{k} Q_{>k+\delta l} \nabla_{x, t}\left[P_{k_{1}} \psi_{1} \nabla^{-1} P_{k_{4}}\left[P_{k_{2}} \psi_{2} P_{k_{3}} \psi_{3}\right]\right] "\right\|_{N[k]} \lesssim 2^{-\nu l} d_{k} \\
& \left\|" P_{k} \nabla_{x, t}\left[P_{k_{1}} Q_{>k_{1}+\delta l} \psi_{1} \nabla^{-1} P_{k_{4}}\left[P_{k_{2}} \psi_{2} P_{k_{3}} \psi_{3}\right]\right] "\right\|_{N[k]} \lesssim 2^{-\nu l} d_{k} \\
& \left\|" P_{k} \nabla_{x, t}\left[P_{k_{1}} \psi_{1} \nabla^{-1} P_{k_{4}}\left[P_{k_{2}} Q_{>k_{2}+\delta l} \psi_{2} P_{k_{3}} \psi_{3}\right]\right] "\right\|_{N[k]} \lesssim 2^{-\nu l} d_{k} \\
& \left\|" P_{k} \nabla_{x, t}\left[P_{k_{1}} \psi_{1} \nabla^{-1} P_{k_{4}} Q_{>k_{4}+\delta l}\left[P_{k_{2}} \psi_{2} P_{k_{3}} \psi_{3}\right]\right] "\right\|_{N[k]} \lesssim 2^{-\nu l} d_{k}
\end{aligned}
$$

Here $\nu>0$ is a constant depending on $\delta$ but not on $\mu$.

Proof. (Lemma 4.14). All of these follow essentially directly from the proof of Proposition 4.6.

The preceding Lemma completes our treatment of the trilinear terms in $(1-$ $\left.\mathbf{P}_{[-\delta l, \delta l]}\right) F\left(\psi_{1}\right)$ since $2^{-\nu l} d_{k} \leq e_{k}$ for $\mu$ (in the definition of $e_{k}$ ) small enough. The quintilinear etc terms in $\left(1-\mathbf{P}_{[-\delta l, \delta l]}\right) F\left(\psi_{1}\right)$ are treated similarly.

ProOF. (Proposition 2.3) This is contained in the preceding proof.

\section{References}

[1] P. Bizon, Comm.Math.Phys.215(2000), 45

[2] D.Christodoulou, A. Tahvildar-Zadeh On the regularity of spherically symmetric wave maps, C.P.A.M., 46(1993), 1041-1091

[3] C.-H. Gu, On the Cauchy problem for harmonic maps defined on twodimensional Minkowski space, Comm. Pure Appl. Math. 33: 727-737, 1980.

[4] S.Klainerman, M.Machedon, Smoothing estimates for null forms and applications, Duke Math.J., 81(1995), 99-133

[5] S.Klainerman, M.Machedon, On the regularity properties of a model problem related to wave maps, Duke Math.J., 87(1997), 553-589

[6] S.Klainerman, I.Rodnianski, On the global regularity of wave maps in the critical Sobolev norm, I.M.R.N. 13(2001), 655-677

[7] S.Klainerman, D.Tataru, On the optimal regularity for the Yang-Mills equations in $\mathbf{R}^{4+1}$, Journal of the American Math. Soc., 12(1999), 93-116

[8] J.Krieger, Global Regularity of Wave Maps in 2 and 3 spatial dimensions, $\mathrm{Ph}$. D. Thesis, Princeton University (2003)

[9] J. Krieger, Global regularity of Wave Maps from $\mathbf{R}^{3+1}$ to surfaces, CMP 238/1-2 (2003), 333-366

[10] J.Krieger, Null-Form estimates and nonlinear waves, Advances in Difftl. Eqns. Vo. 8, no. 10(2003), 1193-1236

[11] J. Krieger, Global regularity of Wave Maps from $\mathbf{R}^{2+1}$ to $\mathbf{H}^{2}$, CMP 250(2004), 507-580

[12] J. Krieger, Global regularity and singularity development for Wave Maps, to appear in "Surveys in Differential Geometry 2007".

[13] A.Nahmod, A.Stefanov, K.Uhlenbeck, On the well-posedness of the wave maps problem in high dimensions, preprint(2001)

[14] I. Rodnianski, T. Tao, Global regularity for the Maxwell-Klein-Gordon equation in high dimensions, preprint.

[15] R. Schoen, S.T. Yau, Lectutres on Difftl. Geometry, International Press(1994), vol.1 
[16] J. Shatah, A. Tahvildar-Zadeh, On the Cauchy Problem for Equivariant Wave Maps, Comm. Pure Appl. Math. 47(1994), 719-754

[17] J. Shatah, A. Tahvildar-Zadeh, On the stability of Stationary Wave Maps, CMP 185(1997), 231-256

[18] T. Sideris, Global existence of harmonic maps in Minkowski space, Comm. Pure Appl. Math. 42(1989), No.1, 1-13

[19] J.Shatah, M. Struwe The Cauchy problem for wave maps, I.M.R.N.11(2002), $555-571$

[20] E.Stein, Harmonic Analysis: Real-Variable Methods, Orthogonality and Oscillatory Integrals, Princeton, NJ, Princeton University Press 1993.

[21] M.Struwe, J.Shatah, Geometric Wave Equations, AMS Courant Lecture Notes 2

[22] C.Sogge, Lectures on nonlinear hyperbolic differential equations

[23] T.Tao, Global regularity of wave maps I, I.M.R.N. 6(2001), 299-328

[24] T.Tao, Global regularity of wave maps II, Comm.Math.Phys.224(2001), 443544

[25] D.Tataru, Local and global results for wave maps I, Comm. PDE 23(1998), 1781-1793

[26] D.Tataru, On global existence and scattering for the wave maps equation, Amer. Journal. Math.123(2001), no.1, 37-77

[27] D. Tataru, Rough solutions for the Wave Maps equation, preprint

[28] K. Uhlenbeck, Connections with $L^{p}$ bounds on curvature, Comm. Math. Phys. 83(1982), 31-42

University of Pennsylvania, Dept. of Mathematics, David Rittenhouse Lab, 209 South 33rd Street, Philadelphia, PA 19104, U.S.A.

E-mail address: kriegerj@math.upenn.edu

Department of Mathematics, Kyoto University, Kyoto 606-8502, Japan

E-mail address: n-kenji@math.kyoto-u.ac.jp 